\title{
Optimal Decomposition and Recombination of Isostatic Geometric Constraint Systems for Designing Layered Materials
}

\author{
T. Baker ${ }^{\mathrm{a}, *}$, M. Sitharam ${ }^{\mathrm{a}}$, M. Wang ${ }^{\mathrm{a}}$, J. Willoughby $^{\mathrm{a}}$ \\ ${ }^{a}$ University of Florida, CSE Building, Gainesville, Florida, U.S.A., 32611
}

\begin{abstract}
Optimal recursive decomposition (or DR-planning) is crucial for analyzing, designing, solving or finding realizations of geometric constraint sytems. While the optimal DR-planning problem is NP-hard even for general 2D bar-joint constraint systems, we describe an $O\left(n^{3}\right)$ algorithm for a broad class of constraint systems that are isostatic or underconstrained. The algorithm achieves optimality by using the new notion of a canonical DR-plan that also meets various desirable, previously studied criteria. In addition, we leverage recent results on Cayley configuration spaces to show that the indecomposable systems - that are solved at the nodes of the optimal DR-plan by recombining solutions to child systems - can be minimally modified to become decomposable and have a small DR-plan, leading to efficient realization algorithms. We show formal connections to well-known problems such as completion of underconstrained systems. Well suited to these methods are classes of constraint systems that can be used to efficiently model, design and analyze quasi-uniform (aperiodic) and self-similar, layered material structures. We formally illustrate by modeling silica bilayers as body-hyperpin systems and cross-linking microfibrils as pinned line-incidence systems. A software implementation of our algorithms and videos demonstrating the software are publicly available online1
\end{abstract}

Keywords: rigidity, geometric constraint solving, configuration spaces, self-similar structures, layered materials

\section{Introduction}

Geometric constraint systems have well-established, mature applications in mechanical engineering and robotics, and they continue to find emerging applications in diverse fields from machine learning to molecular modeling. Solving or realizing geometric constraint systems requires finding real solutions to a large multivariate polynomial system (of equalities and inequalities representing the constraints); this requires double exponential time in the number of variables, even if the type or orientation of the solution is specified. Thus, to realize a geometric constraint system, it is crucial to perform recursive decomposition into locally rigid subsystems (which have finitely many solutions), and then apply the reverse process of recombining the subsystem solutions. With the use of decomposition-recombination (DR-) planning, the complexity is dominated by the size of the largest subsystem that is solved, or recombined, from the solutions of its child subsystems, i.e. the maximum fan-in occurring in a DR-plan. In addition, navigating and analyzing the solution spaces, as well as designing constraint systems with desired solution spaces, leads to the optimal decomposition-recombination (DR-) planning problem [1, 2, 3].

For a broad class of geometric constraint systems, local rigidity is characterized generically as a sparsity and tightness condition of the underlying constraint (hyper)graph [4, 5, 6, 7,. This allows the generic DR-

\footnotetext{
${ }^{1}$ Visit http://cise.ufl.edu/ tbaker/drp/index.html

${ }^{*}$ Corresponding author

Email addresses: tbaker@cise.ufl.edu (T. Baker), sitharam@cise.ufl.edu (M. Sitharam), menghan@cise.ufl.edu (M. Wang), jw5@cise.ufl.edu (J. Willoughby)
} 
planning problem to be stated and treated as a combinatorial or (hyper)graph problem as we do in this paper.

We additionally conjecture that versions of Frontier, a previously given, bottom-up, polynomial time method for obtaining DR-plans with various desired properties [2, 3, 8, 1, in fact generate an optimal DR-plan for non-overconstrained systems.

Naïvely, the optimal DR-plan is used as follows. Each decomposed subsystem - a node of the DR-planis treated and solved as a polynomial system of constraints between its child subsystems. However, even in an optimal DR-plan, there can be arbitrarily many children at a node. In other words, even in the recursive decomposition given by an optimal DR-plan, the size of the maximal indecomposable subsystem could be arbitrarily large. It represents a bottleneck that dictates the complexity of solving or realizing the constraint system [9, 10, 11. We address this problem using the recently developed concept of convex Cayley configuration spaces [12, 13, 14, 15, 16, 17. This allows for even greater reduction of the complexity by realizing large, indecomposable systems in a manner that avoids working with large systems of equations. Specifically, we give an efficient technique for optimally modifying large indecomposable subsystems in a manner that reduces their complexity while preserving desired solutions; the modification ensures a convex Cayley configuration space, and the space can be efficiently searched to find a realization that satisfies the additional constraints of the original system. This optimal modification problem is a generalization of the previously studied problem of optimal completion of underconstrained systems [1, 18].

DR-plans are especially useful for constraint systems that exhibit some level of self-similarity and quasiuniformity, in addition to isostaticity. These properties can be leveraged to further reduce the complexity of both optimal DR-plan construction and recombination. We consider 3 different types of constraint systemswhich we collectively call qusecs - that are used to model, design, and analyze quasi-uniform or self-similar materials. In the remainder of this section, we motivate the materials application and give the contributions and organization of the paper.

\subsection{Introducing Qusecs}

A large class of constraint systems that we call qusecs, a contraction of "quasi-uniform or self-similar constraint system", (a) can be treated combinatorially as described above and (b) occur as independent (isostatic or underconstrained) systems in materials applications. We discuss these next. Some natural and engineered materials can be analyzed by treating them as two dimensional (2D) layers. As illustrated by the examples below, the structure within each layer is often: self-similar ${ }^{2}$ [19, spanning multiple scales; generally aperiodic and quasi-uniform within any one scale; and composed of a few repeated motifs appearing in disordered arrangements. Note that a 2D layer is not necessarily planar (genus 0), it can consist of multiple, inter-constraining planar monolayers. Furthermore, a layer is often either isostatic or underconstrained (not self-stressed/overconstrained, see 2.1 for definitions). These properties, as well as quasi-uniformity, aperiodicity, self-similarity, and layered structure, are natural consequences of evolutionary pressures or design objectives such as stability, minimizing mass, optimally distributing external stresses, and participating in the assembly of diverse and multifunctional, larger structures.

The importance of an optimal DR-plan is particularly evident for a qusecs. The quasi-uniform or selfsimilar properties mean that the decomposition and solution for one subsystem can be used as the decomposition and solution for other subsystems, thus causing further reduction in the complexity of both DR-planning and recombination. This is shown in Figures 3 and 9

Some materials that are readily modeled as qusecs include:

1. Cross-sections of microtubule structures [23] (Figure 1a), e.g., in ciliary membranes and transitions [24].

2. Cross-sections of organic tissue with hierarchical structure, e.g., compact bone and tendon (Figure $1 \mathrm{~b}$.

3. Crosslinked cellulose or collagen microfibril monolayers, e.g., in cell-walls [25, 26], as well as crosslinked actin filaments in the cytoskeleton matrix. See Section 6.

\footnotetext{
${ }^{2}$ In this manuscript we only study finite 2D structures. Self-similarity refers to the result of finitely many levels of hierarchy or subdivision in an iterated scheme to generate self-similar structures.
} 


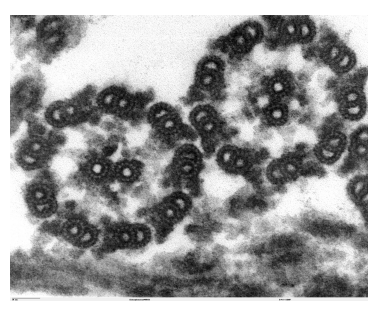

(a)

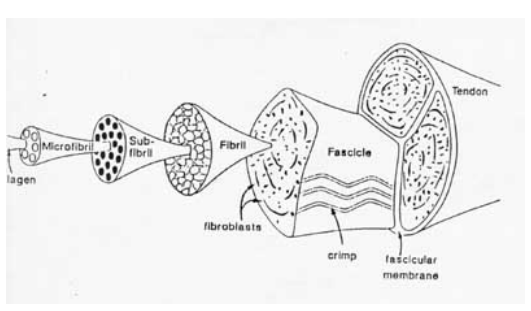

(b)

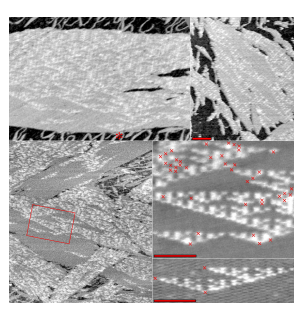

(c)

Figure 1: 1a Cross section of the Chlamydomonas algae axoneme, a cilia composed of microtubules 20. (1b) Cross section of a tendon displaying the hierarchical structure [21. (1c) A DNA array exhibiting the Sierpinski triangle 22 .

4. More recent, engineered examples, including disordered graphene layers [27, 28] sometimes reinforced by microfibrils; and DNA assemblies including a recent Sierpinski gasket [29] (Figure 1c), bringing other self-similar structures [30] within reach.

5. Silica bi-layers 31, glass 32, and materials that behave like assemblies of 2D particles under nonoverlap constraints, i.e. like jammed disks on the plane [33. See Section 5 .

\subsection{Organization and Contributions}

In Section 2, we provide basic definitions in combinatorial rigidity theory, and formalize the new notion of qusecs [9, 10, 11]. In addition, we define DR-plans and what it means for a DR-plan to be complete or optimal. We survey previous work on DR-planning algorithms, discussing other desirable criteria of DR-plans and their relation to the NP-hard optimality property of DR-plans.

In Section 3 , we define a so-called canonical DR-plan and prove a strong Church-Rosser property: all canonical DR-plans for isostatic or underconstrained qusecs are optimal. In so doing, we navigate the NPhardness barrier present in the general form of the DR-planning problem; the canonical DR-plan elucidates the essence of the NP-hardness of finding optimal DR-plans when a system is over-constrained. Furthermore, our optimal/canonical DR-plan satisfies desirable properties such as the previously studied cluster minimality 2. Also in this section, a polynomial time $\left(O\left(n^{3}\right)\right)$ algorithm is provided to find a canonical DR-plan for isostatic bar-joint graphs. While this and the next section focus on bar-joint graphs, the theory is easily extended to other qusecs used to model the abovementioned types of materials, as shown in subsequent sections.

In Section 4, we give a method to deal with the algebraic complexity of recombining the realizations or solutions of child subsystems into a solution of the parent system [9, 10, 11. Specifically, we define the problem of minimally modifying the indecomposable recombination system so that it becomes decomposable via a small DR-plan and yet preserves the original solutions in an efficiently searchable manner. When the modifications are bounded, we obtain new, efficient algorithms for realizing both isostatic and underconstrained qusecs by leveraging recent results about Cayley parameters in [12, 13, 14. (see Sections 4.3 and 4.4. In Section 4.5, we show formal connection to well known problems such as optimal completion of underconstrained systems [18, 1, 34] and to find paths within the connected components.

In Section 5 and 6, we briefly describe applications of the above techniques to modeling, analyzing, and designing specific properties in 2D material layers 35. We explicitly model these materials as qusecs. For Examples 4 and 5, we discuss boundary conditions for achieving various desired properties of body-hyperpin systems. For Example 3, we discuss canonical and optimal DR-plans for pinned line incidence systems [36].

The last Section 7 concludes the paper, and Section 7.1 lists open problems and conjectures. In particular, we conjecture that the methods of Section 3 extend in fact to a large class of (hyper)graphs, formally those with an underlying abstract rigidity matroid in which independence corresponds to some type of sparsity, and maximal independence (rigidity) is a tightness condition. A

Throughout this paper, an asterisk after a formal statement indicates that its proof appears in Appendix 
A software implementation of our algorithms and videos demonstrating the software are publicly available online 3

\section{Preliminaries and Background}

We first give basic definitions and concepts in combinatorial rigidity, leading to a definition of a DR-plan, its properties, and how they relate. The section ends with a discussion of previous work on DR-plans.

\subsection{Geometric Constraint Systems and Combinatorial Rigidity}

In this paper, a geometric constraint system is a multivariate polynomial (usually bilinear or quadratic) system $G(x, \delta)=0$, representing constraints with parameters $\delta$ between geometric primitives in $\mathbb{R}^{2}$ represented collectively as $x \in \mathbb{R}^{n}$. When the type of constraint (system) is fixed, the system is simply represented as $(G, \delta)$, where $G$ is the underlying constraint (hyper)graph $G=(V, E)$ with the vertices $V$ representing the geometric primitives in $\mathbb{R}^{2}$ and (hyper)edges $E$ representing the constraints, each with an associated parameter $\delta$. For example, a bar-joint system or linkage $(G, \delta)$, is a graph $G=(V, E)$ with fixed length bars as edges, i.e. $\delta: E \rightarrow \mathbb{R}$; this represents the distance constraint system $\left\|x_{u}-x_{v}\right\|_{2}=\delta_{u, v}$ for $(u, v) \in E$, where $x_{u} \in \mathbb{R}^{2}$ represents the coordinates of $u \in V$.

In all types of geometric constraint systems we consider in this paper, a Cartesian realization or solution $G(p)$ of $(G, \delta)$ is an assignment of coordinates or Euclidean transformations (poses), $p: V \rightarrow \mathbb{R}^{2}$ or $\mathbb{R}^{3}$, to the vertices of $G$ satisfying the constraints with parameters $\delta$, modulo orientation preserving isometries (Euclidean rigid body motions).

Although the realization space itself depends on the constraint parameters $\delta$, many relevant generic properties of the constraint system $G(x, \delta)$ are defined to be properties of the constraint (hyper)graph $G$ and do not depend on $\delta$ (or they hold for all but a measure zero set of $\delta$ values). Many of these are properties of the Jacobian $\Delta_{x} G(x, \delta)$, often called the appropriate rigidity matrix of $G$ (a matrix of indeterminates). For example, the bar-joint rigidity matrix of the graph $G=(V, E)$ is a matrix of indeterminates representing the Jacobian of the distance map $\left\|x_{u}-x_{v}\right\|_{2}$ for $(u, v) \in E$. The matrix has 2 columns per vertex in $V$ and one row per edge in $E$, where the row corresponding to edge $(u, v)$ contains the 2 coordinate indeterminates for $x_{u}-x_{v}$ (resp. $x_{v}-x_{u}$ ) in the 2 columns for $u$ (resp. $v$ ), i.e. 4 non-zero entries per row.

One important property of a generic constraint system or (hyper)graph ${ }^{4}$ is rigidity, i.e. the realizations or solutions of the corresponding constraint system being generically isolated and zero-dimensional. The result by Asimow and Roth [37] shows a constraint (hyper)graph is rigid if and only if it is generically infinitesimally rigid, i.e. the number of independent rows of its appropriate rigidity matrix is at least the number of columns less the number of rigid body motions, which is 3 for $2 \mathrm{D}$ bar-joint systems.

Geometric constraint systems can also have inequalities in addition to equations, where the parameters in $\delta$ are small intervals rather than exact values. In this case, the definition of rigidity is approximate; the solutions are isolated, small, full-dimensional connected components.

Other generic constraint system or (hyper)graph properties are mentioned here. A constraint (hyper)graph $G$ is independent if its appropriate rigidity matrix of indeterminates has independent rows (i.e. the determinant of some square submatrix is not identically zero). It is isostatic (minimally rigid, wellconstrained) if it is both rigid and independent. It is flexible if it is not rigid, underconstrained if it is independent and not rigid, or overconstrained if it is not independent.

Defining the combinatorial independence of a subset of edges $E^{\prime} \subseteq E$ to be the independence of corresponding rows in the rigidity matrix of indeterminates, we obtain the rigidity matroid of a constraint (hyper)graph $G=(V, E)$. There are various results on combinatorial characterization of independence, rigidity, and rigidity matroids for different types of (hyper)graphs. For bar-joint rigidity matroids, the famous Laman's theorem [4 states that the underlying graph is isostatic if and only if $|E|=2|V|-3$ and $\left|E^{\prime}\right| \leq 2\left|V^{\prime}\right|-3$ for every induced subgraph with at least 2 vertices. The result by Lovasz and Yemini [38]

\footnotetext{
${ }^{3}$ See footnote 1 .

${ }^{4}$ We refer to these as properties of the constraint system or as properties of the underlying (hyper)graph interchangeably
} 


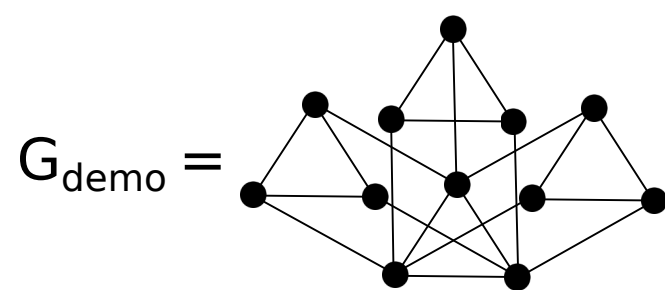

(a)

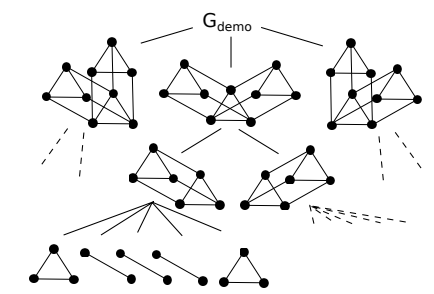

(b)

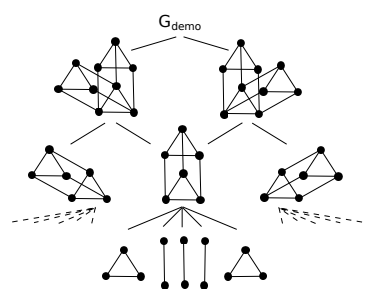

(c)

Figure 2: 2a A graph, $G_{d e m o}$, used to illustrate concepts throughout this and the next section. 2b The complete DR-plan of $G_{d e m o}$, i.e. $\operatorname{ComDRP}\left(G_{d e m o}\right)$. Dashed lines indicate that the children repeat the same pattern as the others shown on this level. The children of triangles (3 edges) are omitted. (2c) The canonical DR-plan of $G_{d e m o}$, which is optimal (see Section 3), i.e. $\operatorname{OptDRP}\left(G_{d e m o}\right)$. The children of triangles are omitted.

shows that all 6-vertex-connected graphs are rigid in the plane. For bar-body rigidity matroids, Tay 6] proved that the underlying multigraph is isostatic if and only if it can be decomposed as 3 edge disjoint spanning trees. White and Whiteley [7] gave the same characterization using a different technique to study the algebraic-geometric conditions of genericity, called pure condition. Lee, Streinu and Theran [39] defined the $(k, l)$-sparsity matroid, where a hypergraph $G$ is called $(k, l)$-sparse if $\left|E^{\prime}\right| \leq k\left|V^{\prime}\right|-l$ for any induced subgraph $\left(V^{\prime}, E^{\prime}\right)$ with at least 2 vertices, and $(k, l)$-tight if it is $(k, l)$-sparse and $|E|=k|V|-l$. In general, given a $d$-uniform hypergraph, a $(k, l)$-sparsity condition is matroidal as long as $l \leq d k-1$.

In this paper, a qusecs is any independent geometric constraint system of one of 3 types: bar-joint (defined formally in 2.1), body-hyperpin (defined formally in Section 5), and pinned line-incidence (defined formally in Section 6).

We note that the remainder of this section and Sections 3 and 4 we only consider bar-joint qusecs and graphs. Relevant formal analogies for the other 2 types of qusecs and (hyper)graphs are given along with their materials applications in Sections 5 and 6.

\subsection{Decomposition-Recombination (DR-) Plans}

Definition 1. The decomposition-recombination (DR-) plan [2] of graph $G, D R P(G)$, is defined as a forest that has the following properties:

1. Each node represents/contains/is a rigid subgraph of $G$.

2. The children $C_{1}, \ldots, C_{N}$ of a node $C$ satisfy $\bigcup_{i=1}^{N} C_{i}=C$.

3. A leaf node is a single edge. A trivial graph is empty or a single vertex. Note that a trivial graph is not isostatic.

4. A root node is a vertex-maximal rigid subgraph of $G$.

A DR-plan is complete if it satisfies an additional property: for a non-leaf node $C$, its children are all of the rigid vertex-maximal proper subgraphs of $C$. This makes Property 2 implicit. We denote a complete $D R$-plan of $G$ as $\operatorname{ComDRP}(G)$.

A DR-plan is optimal if it minimizes the maximum fan-in over all nodes in the tree. The maximum fan-in is called the size of the DR-plan. We denote an optimal DR-plan of G as OptDRP $(G)$.

Remark 2. More than one node (leaf) in a DR-plan forest may represent the same subgraph (vertex) of $G$. For a given graph, there could be exponentially many DR-plans-and even optimal DR-plans-in the size of the graph. A complete DR-plan is unique but may not be (and is usually not) optimal. DR-plans of self-similar graphs are self-similar.

See Figures 2, 3, 4, 5, and 9 for examples of DR-plans and how their properties relate to each other. 


\subsection{Previous Work on DR-Plans}

We now briefly survey existing techniques for detecting rigidity and creating DR-plans of 2D constraint systems. The limitations of these techniques directly motivate the contributions of the next section.

\subsubsection{Finding (Vertex)-Maximal, Generically Rigid Subsystems}

Fast, graph-based algorithms exist (pebble-game [40, 41, 42, 43]), for locating all maximal, generically rigid subsystems (formally defined in 2.1). When the input itself is rigid, these algorithms do nothing, i.e. compute the identity function.

However, both for self-similar or just aperiodic 2D qusecs, it is imperative to recursively decompose rigid systems into their rigid subsystems, down to the level of geometric primitives, in order to understand or design properties at all scales, such as (formally defined in 2.1) rigidity, flexes, distribution of external stresses, boundary conditions for isostaticity, as well as behavior under constraint variations.

\subsubsection{Optimal Recursive Decomposition (DR-Planning)}

Recursive decomposition of geometric constraint systems has been formalized [2, 3] and well-studied 8, 1, 42 as the Decomposition-Recombination (DR-) planning problem (formally defined in Section 2.1). For the abovementioned classes of $2 \mathrm{D}$ qusecs, generic rigidity is a combinatorial property and hence each level of the decomposition should, in principle, be achievable by a graph-based algorithm without involving the geometric information in the constraint system. Since many such decompositions can exist for a given constraint system, criteria defining desirable or optimal DR-plans and DR-planning algorithms were given in [2].

However, for overconstrained 2D qusecs, even when restricted to bar-joint systems, the optimal DRplanning problem was shown to be NP-hard [8, 1]. The NP-hardness of the optimal DR-planning problem for $2 \mathrm{D}$ bar-joint graphs is partly the consequence of possibly exponential number of DR-plans. On the other hand, although the complete DR-plan is unique it could have large average fan-in and exponentially many nodes making it far from optimal.

\subsubsection{DR-plans for Special Classes and with Other Criteria}

For a special class of 2D qusecs, namely tree-decomposable systems [44, 45, 46] common in computer aided mechanical design (which includes ruler-and-compass and Henneberg-I constructible systems), all DR-plans turn out to be optimal. This satisfies the Church-Rosser property, leading to highly efficient DR-planning algorithms. For general 2D qusecs, alternate criteria were suggested such as cluster minimality requiring parent systems to have a minimal set of at least 2 rigid proper subsystems as children (i.e. the union of no proper subset of size at least 2 child subsystems forms a rigid system); and proper maximality, requiring child subsystems to be maximal rigid proper subsystems of the parent system. See 2.1 for formal definitions.

While polynomial time algorithms were given to generate DR-plans meeting the cluster minimality criterion [8, no such algorithm is known for the latter criterion.

\section{Main Result: Canonical DR-Plan, Optimality, and Algorithm}

\subsection{Canonical DR-Plan}

In this section, we define a canonical plan to capture those aspects of an optimal DR-plan that mimic the uniqueness of a complete DR-plan, and we show that the nonunique aspects do not affect optimality for independent (underconstrained or isostatic) graphs. Furthermore, we give an efficient $O\left(n^{3}\right)$ algorithm to find the canonical DR-plan of any independent graph. The definition is as follows:

Definition 3. The canonical DR-plan of a graph $G$ satisfies the following three properties: (1) it is a DRplan of $G$; (2) children are rigid vertex-maximal proper subgraphs of the parent; and (3) if all pairs of rigid vertex-maximal proper subgraphs intersect trivially then all of them are children, otherwise exactly two that intersect non-trivially are children. 
In this section and in section 4 , any reference to a graph $G$ is assumed to be isostatic (i.e. well-constrained or $(k, l)$-tight).

Definition 3 gives the canonical DR-plan a surprisingly strong Church-Rosser property, which is made explicit in Theorem 4, the main result of this section.

Theorem 4. A canonical DR-plan exists for a graph $G$ and any canonical DR-plan is optimal if $G$ is independent.

Proof. We show the existence of a canonical DR-plan by constructing it as follows:

Begin with $\operatorname{ComDRP}(G)$ of a rigid $2 \mathrm{D}$ bar-joint graph $G$, for all nodes $C$ with children $C_{1}, \ldots, C_{N}$ retain children nodes according to the following rules:

1. If $C_{i} \cap C_{j}$ is trivial then retain all $C_{1}, \ldots, C_{N}$ as children.

2. If $C_{i} \cap C_{j}$ is rigid then select any two out of $C_{1}, \ldots, C_{N}$ as children.

This directly satisfies Properties (2) and (3) of a canonical DR-plan (see Definition 3), because all the nodes in $\operatorname{ComDRP}(G)$ are rigid vertex-maximal proper subgraphs, which we shorten to clusters. To show Property (1) holds (that this constitutes a DR-plan): for Case 1 above, since we start with a complete DR-plan, if we preserve all the children it is still a DR-plan; for Case 2 above, we know that the union must be rigid as well and it cannot be anything other than $C$, otherwise we would have found a larger rigid proper subgraph of $C$, contradicting vertex-maximality.

Note that if we begin with an isostatic graph, "rigid" can be replaced with "isostatic" throughout the construction and preserve the above properties. The rigid proper subgraphs of an isostatic graph must be isostatic themselves.

Next we show that a canonical DR-plan is optimal.

First, note that any DR-plan $R$ without the Property (2) of a canonical DR-plan can always be modified (by introducing intermediate nodes) to satisfy Property (2) without increasing the max fan-in, since any rigid proper subgraph of a graph $C$ (a child of node $C$ of the DR-plan $R$ ) is the subgraph of some cluster of $C$. Thus without loss of generality, we can assume that an optimal DR-plan satisfies Property (2) of a canonical DR-plan.

The proof of optimality of a canonical DR-plan is by induction on its height. The base case trivially holds for canonical DR-plans of height 0, i.e. for single edges. The induction hypothesis is that canonical DR-plans of height $t$ are optimal for the root node. For the induction step consider a canonical DR-plan $R$ of height $t+1$ rooted at a node $C$. Notice that $R$ represents a canonical DR-plan $R(C)$ for the graphs $C$ corresponding to each of its descendant nodes. Thus, from the induction hypothesis, we know that the $R\left(C_{i}\right)$ is optimal for $C_{i}$.

Thus it is sufficient to demonstrate a set of nodes $S$ that must be present in any DR-plan $R$ for $C$ that satisfies Property (2), including a known optimal one; and furthermore, for any such DR-plan $R$, either (Claim 1) $S$ must be the set of children of $C$; or (Claim 2) for all the ancestors $A$ of $S, R$ has the minimum possible fan-in of 2 .

We show the two claims below. The first claim is that for a node $C$ whose clusters have trivial pairwise intersections, any DR-plan of $C$ that satisfies Property (2) must also satisfy Property (3) at $C$, i.e. the set of children $S$ of $C$ consists of all clusters of $C$. Because this is the only choice, it is the minimum fan-in at $C$ for any DR-plan for $C$ with Property (2), including a known optimal one. The second claim shows that in the case of nodes $C$ whose rigid, vertex-maximal proper subgraphs have non-trivial pairwise intersections, every canonical DR-plan of $C$ that uses any possible choice of two such subgraphs of $C$ as children results in a minimum possible fan-in of 2 in the ancestor nodes $A$ leading to the same maximal antichain $S$ of descendants $D$ of $C$. The antichain is maximal in the partial order of rigid subgraphs of $C$ under containment. I.e. $S$ satisfies the property that every proper vertex-maximal rigid subgraph of $C$ is a superset of some $D$ in $S$; this follows from properties of maximal antichains that no element of $S$ is contained in the union of other elements of $S$; and the union of elements of $S$ is $C$. Thus any DR-plan that satisfies Property (2) and hence contains two or more of the rigid vertex-maximal proper subgraphs of $C$ as children must also contain every element of $S$. The two claims complete the proof that every canonical DR-plan is optimal. 
Claim 1: A set of clusters $C_{1}, \ldots, C_{N}$ whose pairwise intersection is trivial, must be children of $C$ in an optimal DR-plan.

We prove this claim by showing that the union of no subset of the children can be $C$, thereby requiring all of them to be included as children.

We prove by contradiction. Assume to the contrary that the strict subset $S \subsetneq\{1, \ldots, N\}$ such that $U=\bigcup_{i \in S} C_{i}$ is isostatic. If $U \neq C$, then we found a larger proper subgraph contradicting vertex-maximality of the $C_{i}$. So, it must be that $U=C$. However, since $C_{i} \cap C_{j}$ is trivial then for $k \notin S$ we know, by Lemma 6. Item 3, $U \cap C_{k}$ must be one or more trivial, i.e. disconnected vertices. By definition of a DR-plan, $C_{k}=C \cap C_{k}$ and we know that $U=C$ so $C_{k}=U \cap C_{k}$. Thus, $C_{k}$ is (i) a collection of disconnected vertices, and (ii) an isostatic subgraph of $C$, which is impossible. As $C$ is isostatic, this means the union of no proper subset of $C_{1}, \ldots, C_{N}$ is isostatic, nor is it equal to $C$, proving Claim 1.

Furthermore, since a canonical DR-plan has nodes with proper rigid vertex-maximal subgraphs as children, if, as in this case, their pairwise intersection is trivial, it follows that any node has at most as many children as a DR-plan without this restriction, because the union of the children must contain all edges of the parent. Therefore, the canonical DR-plan is the optimal choice in this case of trivial intersections.

Claim 2: If some pair in the set of child clusters $C_{1}, \ldots, C_{N}$ of $C$ has an isostatic (nontrivial) intersection, then choosing any two as children (minimum possible fan-in) will result in the same maximal antichain of descendants of $C$.

To prove Claim 2, notice that if $C_{i} \cap C_{j}$ is isostatic, then, by Observation $5, C_{i} \cup C_{j}$ is also isostatic. This means that, by Lemma 6. Point 2, the union of any two children of $C$ is $C$ itself. Thus, any two children can be chosen to make a canonical DR-plan and that is the minimum fan-in possible for a node of the DR-plan.

However, to guarantee that any two are the optimal choice, it must ensure minimum fan-in over all descendants leading up to a common maximal antichain $S$ of subgraphs.

To prove this holds, take the set $[N]=\{1, \ldots, N\}$, and denote $I=\bigcap_{k \in[N]} C_{k}$ and $R_{k}=C \backslash C_{k}$. Suppose $C_{i}$ and $C_{j}$, where $i \neq j$, are the children. For convenience, we will assume all subgraphs are induced subgraphs of $C$. We know that $C=I \cup \bigcup_{k \in[N]} R_{k}$ and $C_{i}=I \cup \bigcup_{k \in[N] \backslash\{i\}} R_{k}$. The isostatic vertexmaximal subgraphs of $C_{i}$ are $\left(I \cup \bigcup_{k \in[N] \backslash\{i, 1\}} R_{k}\right), \ldots,\left(I \cup \bigcup_{k \in[N] \backslash\{i, i-1\}} R_{k}\right),\left(I \cup \bigcup_{k \in[N] \backslash\{i, i+1\}} R_{k}\right), \ldots,(I \cup$ $\left.\bigcup_{k \in[N] \backslash\{i, N\}} R_{k}\right)$ all of whose pairwise intersections are isostatic subgraphs. So any two of these are viable children for $C_{i}$. This continues for $N-1$ levels, always with fan-in of two (the minimum possible), at which point every descendant of $C$ is some $I \cup R_{k}$ for $k \in[N]$, with every $k$ appearing at least once. At the last level, there are exactly two rigid proper vertex-maximal subgraphs, and hence a unique choice of pair of children. Thus, regardless of the sequence of choices of $C_{i}$ and $C_{j}$, and of their descendants at each level, the DR-plan has the optimal fan-in of two for every node for $N$ levels, and the collection of last level nodes contain the same maximal antichain of subgraphs (for all choices).

This proof of this theorem relies on the following crucial observation and lemma. These will be used again in the application sections (5) and 6) of the paper, with modifications to work for other types of qusecs.

Observation* 5. If $F_{i}$ and $F_{j}$ are non-empty isostatic graphs then the following hold:

(1) $F_{i} \cup F_{j}$ is not trivial; (2) $F_{i} \cup F_{j}$ is underconstrained if and only if $F_{i} \cap F_{j}$ is trivial; (3) $F_{i} \cup F_{j}$ is isostatic if and only if $F_{i} \cap F_{j}$ is isostatic; and (4) $F_{i} \cap F_{j}$ is not underconstrained.

The following key properties hold at the nodes of a canonical DR-plan.

Lemma* 6. Let $C$ be an isostatic node of a canonical DR-plan, with distinct children $C_{1}, C_{2}, \ldots, C_{m}$. Assume $i \neq j$. Then

1. $C_{i} \cup C_{j}$ is isostatic if and only if $C_{i} \cup C_{j}=C$.

2. If $C_{i} \cup C_{j}$ is isostatic, then $\forall k: C_{i} \cup C_{k}$ is isostatic. Alternatively, if $C_{i} \cup C_{j}=C$, then $\forall k: C_{i} \cup C_{k}=C$.

3. If $C_{i} \cap C_{j}$ is trivial, then $\forall k: C_{i} \cap C_{k}$ is trivial.

Remark 7. The first item in the above lemma generalizes to the union of any number of children, $C_{1}, \ldots, C_{k}$, resulting in the desirable property of cluster minimality (defined in [2] and in Section [2.3) holding for canonical-optimal DR-plans. 


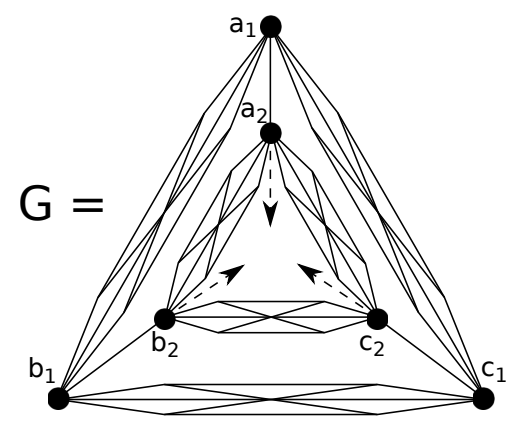

(a)

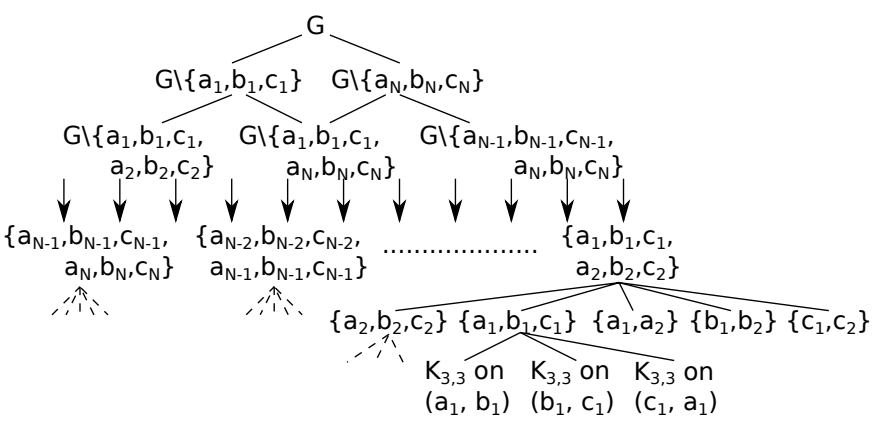

(b)

Figure 3: 3a A sequence of doublets $\left(C_{2} \times C_{3}\right)$ intersecting on triangles, where the edges of the triangles are replaced by $K_{3,3}$ 's. This pattern continues inwards for a total of $N$ triangles, indicated by the dashed arrows. (3b The canonical DR-plan of $G$, drawn as a DAG. $G \backslash\left\{a_{i}, b_{i}, c_{i}\right\}$ is shorthand for $G$ difference those nodes and all of the nodes in the corresponding $K_{3,3}$ subgraphs. Below the third level, the obvious pattern continues until only the individual doublets are present (fourth level) with the ellipses indicating the remaining doublets between those shown. Decomposition of one of these doublets is shown. The dashed lines indicated that this exact decomposition (of the similar nodes on the level) is repeated. Further decomposition of $K_{3,3}$ subgraphs into the separate 9 edges is omitted from the figure.

Example 8 (DR-plan for self-similar structure). This example details the decomposition of the graph in Figure 3. the canonical DR-plan of $G$. It begins with the whole (isostatic) graph as the root. The graph $G$ has only two isostatic vertex-maximal subgraphs: $G$ without the outermost triangle composed of $K_{3,3}$ graphs (triangle 1 ) and $G$ without the inner triangle (triangle $N$ ). These intersect on $G$ without triangle 1 and $N$ which is clearly isostatic. As explained in the proof of Theorem 4, since there are only 2 possible children, their intersection must be a node 2 levels below the parent. As expected, it is on the third level, as a child of both of $G$ 's children.

Both of $G$ 's children are similar to $G$, but containing only $N-1$ triangles. Therefore, the canonical DR-plans of these children follow the same pattern. This continues downward until the individual doublets are reached (there will be multiple occurrences of the same doublets at this level, but they can be represented as the same node in a DAG).

Further decomposition of one of these doublets is shown. The three edges between the triangles and the triangles themselves all intersect trivially pairwise. By Theorem 4, part 1, they must all be children in the DR-plan. Similarly, the triangles decompose into their three trivially intersecting $K_{3,3}$ 's. Then the $K_{3,3}$ subgraphs decompose into their separate 9 edges.

The self-similar nature of this graph is evident in the canonical DR-plan. Many structures are repeated throughout the DR-plan, allowing for shared computation in both decomposition and recombination.

\subsection{Algorithm}

Theorem 9. There exists an $O\left(|V|^{3}\right)$ algorithm to find a canonical DR-plan for an input graph.

Proof. We first describe the algorithm. The first step of the algorithm, which we call $\operatorname{CanDRP}(G)$, is finding the isostatic vertex-maximal proper subgraphs (clusters) of the input isostatic graph $G$. One of many ways to do this is by first dropping arbitrary edge $e$ from the edge set and running the pebble game algorithm [40] on this subgraph, which is $O\left(|V|^{2}\right)$. The output of this will be a list of cluster-candidates. The next step is to isolate the set of true-clusters by running the Frontier algorithm 3$][8,(O(|V|))$ on each candidate along with edge $e$, to find the minimal subgraph $D$ that contains the candidate and $e$. If $D=G$, the candidate is moved (before adding $e$ ) to the true-cluster list and removed from the candidate-cluster list. If $D \subsetneq G$, all candidates that are subgraphs of $D$ are removed from the candidate-cluster list and $D$ is added back to the list. The next candidate is considered until all candidates are exhausted. 


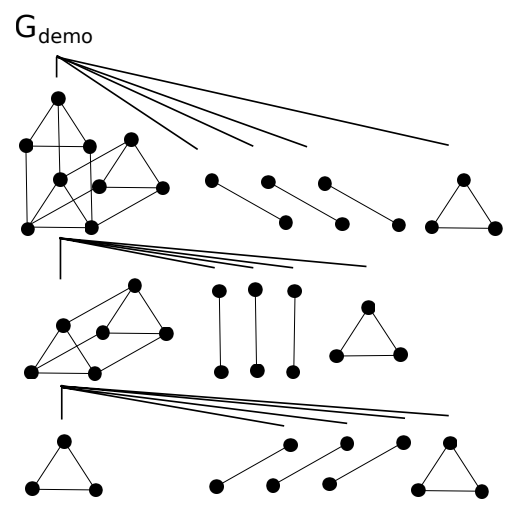

Figure 4: The sequential canonical DR-plan of $G_{\text {demo }}$ from Figure 2a, which is optimal (as explained in the proof of Theorem 9). The children of the triangle are omitted. Compare to to the typical canonical DR-plan shown in Figure 2c. Also, note that the bottom-left node, the triangle, is the intersection of the 3 children of $G_{\text {demo }}$ in $\operatorname{ComD} R P\left(G_{d e m o}\right)$, shown in Figure $2 \mathrm{~b}$

The next step is to check the intersection of any two of the true-components. If the intersection is trivial, the entire list becomes the children, and the algorithm is recursively applied to each child. If the intersection is non-trivial (necessarily isostatic), the decomposition is computed down to $I=\bigcap_{k=1}^{N} C_{k}$. (This is discussed in detail in the proof of Theorem 4). We construct a DR-plan whose node set is that of a canonical/optimal DR-plan. Begin with the parent $C$. Its children are $S_{1}$ and $\operatorname{CanDRP}\left(T_{1}\right)$, where $S_{1}=C \backslash R_{1}$ and $T_{1}$ is $R_{1}$, together with all incident edges. Observe that these edges are incident on vertices from $I$ and that $T_{1}$ is underconstrained so its DR-plan is a forest where each root becomes a child of $C$. The children of $S_{1}$ are $S_{2}$ and $\operatorname{CanDRP}\left(T_{2}\right)$, where $S_{2}=C \backslash\left(R_{1} \cup R_{2}\right)$ and $T_{2}$ is $R_{2}$ together with all incident edges. The process iterates. At $N$ levels down, the children are $S_{N}=I$ and $\operatorname{Can} D R P\left(T_{N}\right)$, where $T_{N}$ is $R_{N}$ together with all incident edges. Apply the algorithm recursively to both children. Thus we obtain an efficient method for the case of DR-plan nodes whose rigid proper vertex-maximal subgraphs have non-trivial intersections, avoiding recomputation of the isostatic components of $C$. See Figure 4 for an example.

To summarize the above step of the algorithm: if the intersection is trivial, recursively apply $\operatorname{CanDRP}$ to the entire list of true-components and set the resulting trees as the children. Else, the intersection is non-trivial, and the children of the node are $\operatorname{CanDRP}\left(C_{1}\right)$ and $\operatorname{CanDRP}(T)$, where $C_{1}$ is the first truecomponent of the parent $C$ (chosen arbitrarily, could be any child) and $T$ is the underconstrained graph formed by $C \backslash C_{1}$ together with all incident edges and the associated nodes in $C_{1}$.

The DR-plan output by this algorithm is a tree where each node is a distinct subgraph. The leaves of this tree are the edges of the starting graph $G$. Therefore, the number of nodes in the tree is $O(|E|)$ which, for isostatic input graphs, is $O(|V|)$ and the complexity of the algorithm is $O\left(|V|^{3}\right)$.

\subsection{Overconstrained Graphs and NP-Hardness of Optimal DR-Planning}

For overconstrained (not independent) graphs, a canonical DR-plan is still well-defined. However, it may be far from optimal. The proofs of Theorem 4, Observation 5, and Lemma 6 all fail for overconstrained graphs. It is important to note that, regardless whether the graph is overconstrained, if every node in a canonical DR-plan $R$ has clusters whose pairwise intersection is trivial, then the DR-plan is the unique one satisfying Property (2), and since we know that there is an optimal DR-plan that satisfies Property (2), $R$ is in fact optimal. The problem arises when some node in a DR-plan has clusters whose pairwise intersection is non-trivial. In this case, an arbitrary choice of a pair of clusters as children of an overconstrained node in a canonical DR-plan may not result in an optimal DR-plan. This is in contrast to independent graphs, which, as shown in Theorem 4, exhibit the strong Church-Rosser property that any choice yields an optimal DRplan. A good source of examples of overconstrained graphs with canonical DR-plans that are not optimal are graphs whose cluster-minimal DR-plans that are not optimal. The example shown in Figure 5 is a 


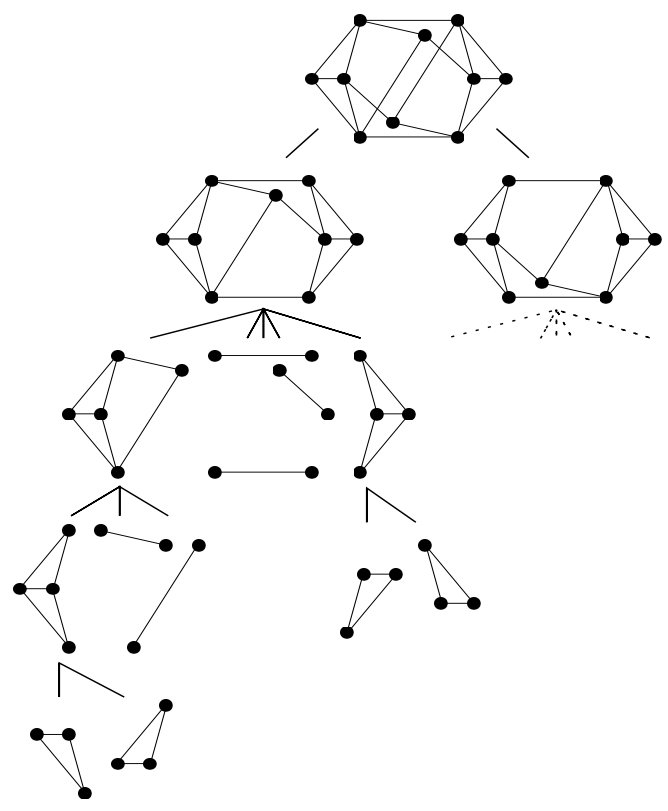

(a)

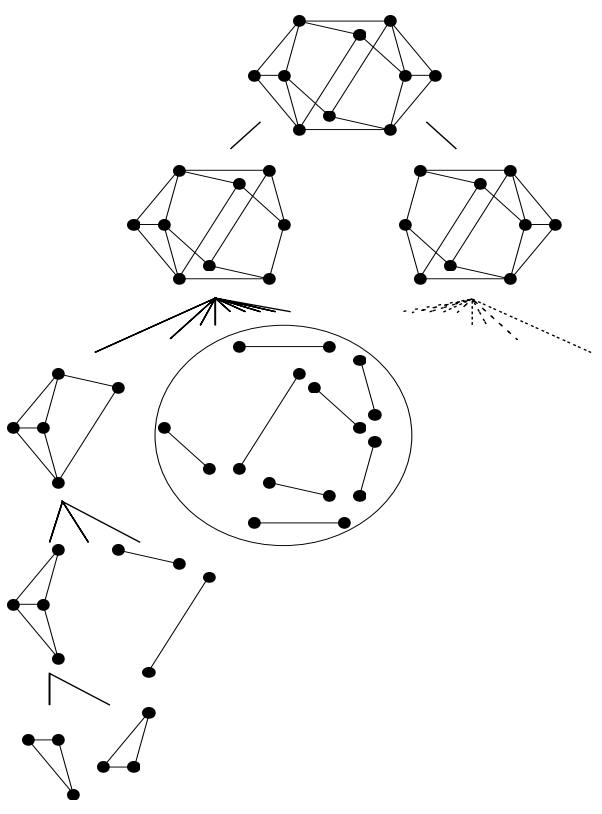

(b)

Figure 5: Both figures are canonical and cluster-minimal DR-plans of the same singly overconstrained rigid graph. Decomposition of triangles are omitted and dashed lines indicate a decomposition similar to the other nodes on the same level. (5a) is an optimal DR-plan, with a fan-in of 5. (5b) has a fan-in of 9 and is non-optimal, shown by the preceding counter-example.

canonical, cluster-minimal DR-plan that is not optimal; an optimal DR-plan is also shown in the figure. The root cause of the NP-hardness is encapsulated in this figure: because the different choices of vertexmaximal subgraphs for overconstrained input do not incur the same fan-in, finding the optimal DR-plan becomes a search problem with a combinatorial explosion of options.

As mentioned earlier, the Modified Frontier algorithm version given in [8] runs in polynomial time and finds a cluster-minimal DR-plan for any graph. Similarly, the algorithm given above finds a canonical DRplan also for any input graph. However neither of these DR-plans may be optimal for overconstrained graphs as shown in Figure 5.

While the canonical DR-plan is optimal only if the input graph is independent, when there are only $k$ overconstraints for some fixed $k$, we can still find the optimal DR-plan using a straightforward modification of the above algorithm. However, the time complexity is exponential in $k$.

This exponential growth of time complexity for overconstrained graphs is in fact captured in the proof of NP-hardness of optimal DR-planning in [1, 8].

\section{Recombination and Problem Relationships}

In this section, we consider the optimal recombination problem of combining specific solutions of subsystems in a DR-plan into a solution of their parent system $I$ (without loss of generality, at the top level of the DR-plan). In the case of isostatic qusecs, the parent system $I$ is isostatic (the root of the DR-plan), and we seek solution(s) (among a finite large number of solutions) with a specific orientation or chirality. In the case of underconstrained qusecs the subsystems are the multiple roots of the DR-plan, the parent system $I$ is underconstrained, and we typically seek an efficient algorithmic description of connected component(s) of solutions with a specific orientation or chirality.

The main barrier in recombination when given an optimal DR-plan (of smallest possible size or max fanin) for a system $S$, is that the number of children of the root (resp. number of roots of the DR-plan) - and 
correspondingly the size and complexity of the (indecomposable) algebraic system $I$ to be solved - could be arbitrarily large as a function of the size of $S$. This difficulty can persist even after optimal parametrization of the indecomposable system $I$ as in 9 to minimize its algebraic complexity.

\subsection{Previous Work}

We now briefly survey existing techniques for handling the complexity of recombination of DR-plans for qusecs. The limitations of these techniques directly motivate the contributions in this section.

\subsubsection{Optimal Recombination and Solution Space Navigation}

For the entire DR-plan, finding all desired solutions is barely tractable even if recombination of solved subsystems is easy for each indecomposable parent system in the DR-plan. This is because even for the simplest, highly decomposable systems with small DR-plans, the problem of finding even a single solution to the input system at the root of the DR-plan is NP-hard [47] and there is a combinatorial explosion of solutions [4]. Typically, however, the desired solution has a given orientation type, in which case, the crux of the difficulty is concentrated in the algebraic complexity of (re)combining child system solutions to give a solution to the parent system at any given level of the DR-plan. For fairly general 3D constraint systems, there are algorithms with formal guarantees that uncover underlying matroids to combinatorially obtain an optimal parameterization to minimize the algebraic complexity of the indecomposable parent (sub)systems that occur in the DR-plan [9, 10, 11, provided the DR-plan meets some of the abovementioned criteria.

However, the generality of these algorithms trades-off against efficiency, and, despite the optimization, the best algorithms can still take exponential time in the number of child subsystems (which can be arbitrarily large even for optimal DR-plans) in order to guarantee all solutions of a given orientation type, even for a single (sub)system in a DR-plan. They are prohibitively slow in practice. We note that, utilizing the DR-plan and optimal recombination as a principled basis, high performance heuristics and software exists [49] to tame combinatorial explosion via user intervention.

\subsubsection{Configuration Spaces of Underconstrained Systems}

For underconstrained 2D bar-joint and body-hyperpin qusecs obtained from various subclasses of treedecomposable systems, algorithms have been developed to complete them into isostatic systems [18, 1, 34, 12, and to find paths within the connected components [13, 50] of standard Cartesian configuration spaces. Most of the algorithms with formal guarantees leverage Cayley configuration space theory [12, 13, 14, to characterize subclasses of graphs and additional constraints that control combinatorial explosion, and provide faithful bijective representation of connected components and paths. These algorithms have decreasing efficiency as the subclass of systems gets bigger, with highest efficiency for underlying partial 2-tree graphs (alternately called tree-width 2 , series-parallel, and $K_{4}$ minor avoiding), moderate efficiency for 1 degree-offreedom (dof) graphs with low Cayley complexity (which include common linkages such as the Strandbeest, Limacon, and Cardioid), and decreased efficiency for general 1-dof tree-decomposable graphs. While software suites exist [51, 52, 53, 54], no such formal algorithms and guarantees are known for non-tree-decomposable systems.

\subsection{Optimal Modification for Recombination}

In the following, we formulate the problem of optimal modification of an indecomposable algebraic system $I$ at some node of a (possibly optimal) DR-plan into a decomposable system with a small DR-plan (low algebraic complexity). Leveraging recent results on Cayley configuration spaces, our approach to the optimal modification problem achieves the following:

(a) Small DR-plan. We obtain a parameterized family of systems $I_{\lambda_{F}}$-one for each value $\lambda_{F}$ for the parameters $F$, all of which have small DR-plans. Thus, given a value $v$ for $\lambda_{F}$, the system $I_{v}$ can potentially be solved or realized easily once the orientation type of the solution is known (when the DR-plan size is small enough).

(b) Solution preservation. Moreover, the union of solution spaces of the systems in the family $I_{\lambda_{F}}$ is guaranteed to contain all of $I$ 's solutions. 
(c) Efficient search. Finally, the so-called Cayley or distance parameter space $\lambda_{F}$ is convex or otherwise easy to traverse in order to search for I's solution (or connected component) of the desired orientation type. For the case when the modification (number of Cayley parameters) is bounded, this approach provides an efficient algorithm for recombination. We first define the decision version of the problem of optimal modification for decomposition. The standard optimization versions are straightforward.

Optimal Modification for Decomposition (OMD) Problem. Given a generically independent graph $G=(V, E)$ with no non-trivial proper isostatic subgraph (indecomposable) and 2 constants $k$ and $s$, does there exist a set of at most $k$ edges $E_{1}$ and a set of non-edges $F$ such that the modified graph $H=$ $\left(V, E \backslash E_{1} \cup F\right)$ has a DR-plan of size at most $s$ ? The $\mathrm{OMD}_{k}$ problem is OMD where $k$ is a fixed bound (not part of the input). We say that such a tuple $(G, s)$ is a member of the set $\mathrm{OMD}_{k}$. We loosely refer to graphs $G$ as OMD with appropriately small $k$ and $s$ or $\mathrm{OMD}_{k}$ with appropriately small $s$.

It is immediately clear that indecomposable graphs $G$ that belong in $\mathrm{OMD}_{k}$ for small $k$ and $s$ lend themselves to modification into decomposable graphs satisfying Criteria (a) and (b) above. However, it is not clear how Criterion (c) is met by OMD graphs. Before we consider this question, we discuss previous work on recombination of DR-plans.

\subsection{Using Convex Cayley Configuration Spaces}

Next we provide the necessary background to describe a specific approach for achieving the requirements (a)-(c) mentioned above, by restricting the class of reduced graphs $G^{\prime}=G \backslash E_{1}$ and their isostatic completions $H$ in the above definition of the OMD problem, and using a key theorem of Convex Cayley configuration spaces [12. This theorem characterizes the class of graphs $H$ and non-edges $F$ (Cayley parameters), such that the set of vectors $\lambda_{F}$ of attainable lengths of the non-edges $F$ is always convex for any given lengths $\delta$ for the edges of $H$ (i.e. over all the realizations of the bar-joint constraint system or linkage $(H, \delta)$ in 2 dimensions). This set is called the (2-dimensional) Cayley configuration space of the linkage $(H, \delta)$ on the Cayley parameters $F$, denoted $\Phi_{F}(H, \delta)$ and can be viewed as a "projection" of the cartesian realization space of $(H, \delta)$ on the Cayley parameters $F$. Such graphs $H$ are said to have convexifiable Cayley configuration spaces for some parameters $F$ (short: $H$ is convexifiable).

To state the theorem, we first have to define the notion of 2-sums and 2-trees. Let $H_{1}$ and $H_{2}$ be two graphs on disjoint sets of vertices $V_{1}$ and $V_{2}$, with edge sets $E_{1}$ and $E_{2}$ containing edges $(u, v)$ and $(w, x)$ respectively. A 2-sum of $H_{1}$ and $H_{2}$ is a graph $H$ obtained by taking the union of $H_{1}$ and $H_{2}$ and identifying $u=w$ and $v=w$. In this case, $H_{1}$ and $H_{2}$ are called 2-sum components of $H$. A minimal 2-sum component of $H$ is one that cannot be further split into 2-sum components. A 2-tree is recursively obtained by taking a 2-sum of 2-trees, with the base case of a 2-tree being a triangle. A partial 2-tree is a 2-tree minus some edges. Partial 2-trees have an alternate characterization as the graphs that avoid $K_{4}$ minors, and are also called series-parallel graphs.

Theorem 10. [12] $H$ has a convexifiable Cayley configuration space with parameters $F$ if and only if for each $f \in F$ all the minimal 2-sum components of $H \cup F$ that contain both endpoints of $f$ are partial 2-trees. The Cayley configuration space $\Phi_{F}(H, \delta)$ of a bar-joint system or linkage $(H, \delta)$ is a convex polytope. When $H \cup F$ is a 2-tree, the bounding hyperplanes of this polytope are triangle inequalities relating the lengths of edges of the triangles in $H \cup F$.

The idea of our approach to achieve the criteria (a)-(c) begins with the following simple but useful theorem.

Theorem 11. Given an indecomposable graph $G$, let $G^{\prime}$ be a spanning partial 2-tree subgraph in $G$ with $k$ fewer edges than $G$. Then $(G, 2)$ belongs in the set $O M D_{k}$.

Proof. The proof follows from the fact that 2-trees are well decomposable and have simple DR-plans of size 2. We know that $G$ can be reduced by removing $k$ edges to create a partial 2 -tree $G^{\prime}$ which can then be completed to an (isostatic) 2-tree by adding some set of non-edges $F$. Thus the modified graph $H=G^{\prime} \cup F$ has a DR-plan of size 2, proving the theorem. 


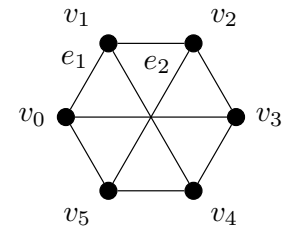

(a)

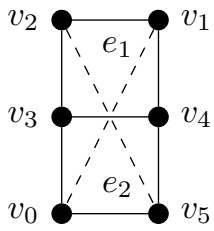

(b)

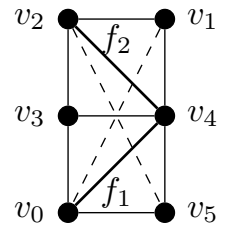

(c)

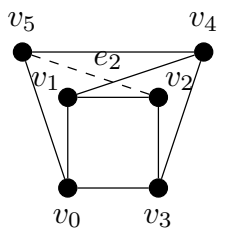

(d)

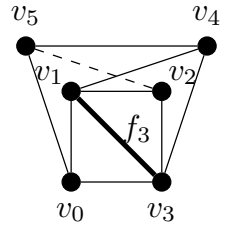

(e)

Figure 6: 6a The $K_{3,3}$ with two labeled edges, $e_{1}$ and $e_{2}$. (6b) The $K_{3,3}$ with $e_{1}$ and $e_{2}$ removed (dashed lines) and rearranged to illustrate that it is now a partial 2-tree. (6c) The $K_{3,3}$ with $\left\{e_{1}, e_{2}\right\}$ removed and $\left\{f_{1}, f_{2}\right\}$ (bold lines) added to make a 2 -tree, showing that the $K_{3,3}$ is at least $\mathrm{OMD}_{2}$. (6d) The $K_{3,3}$ with only $e_{2}$ removed (dashed line). (6e) The $K_{3,3}$ with $e_{2}$ removed and $f_{3}$ (bold line) added to make a low Cayley complexity graph, showing that the $K_{3,3}$ is $\mathrm{OMD}_{1}$.

We refer to such graphs $G$ in short as $k$-approximately convexifiable, where the reduced graphs $G^{\prime}$ and isostatic completions $H$ are convexifiable. As observed earlier, since graphs such as $G$ are in $\mathrm{OMD}_{k}$, Criteria (a) and (b) are automatically met for small enough $k$. Criterion (c) is addressed as described in the following efficient search procedure which clarifies the dependence of the complexity on the number and ranges of Cayley parameters $F$.

Theorem* 12 (Efficient search). For an indecomposable, $k$-approximately convexifiable graph $G=(V, E)$, let $G^{\prime}=\left(V, E^{\prime}=E \backslash D\right)$ be a spanning partial 2-tree subgraph where $|D| \leq k$. Let $F$ be a set of non-edges of $G$ such that $H=\left(V, E^{\prime} \cup F\right)$ is a 2-tree. Each solution p (or connected component of a solution space) of $(G, \delta)$ of an orientation type $\sigma_{p}$ can be found in time $O(\log (W))$ where $W$ is the number of cells of desired accuracy (discrete volume) of the convex polytope $\Phi_{F}\left(G^{\prime}, \delta_{E}^{\prime}\right)$. The (discrete) volume $W$ is exponential in $|F|$ and polynomial in the (discrete range) of the parameters in $F$.

Note: A major advantage of the convex Cayley method is that it is completely unaffected when $\delta$ are intervals of values rather than exact values [12.

Example 13 (Using Cayley configuration space). A graph $G=K_{3,3}$ cannot be decomposed into any nontrivial isostatic graphs, i.e. its DR-plan has a root and 9 children corresponding to the 9 edges. Solving or recombining the system $(G, \delta)$ corresponding to the root of this DR-plan involves solving a quadratic system with 8 equations and variables. Instead of simultaneously solving this system, we could instead use the fact that $G=K_{3,3}$ is in $O M D_{2}$ : remove the edges $e_{1}, e_{2}$ in Figure 6 to give a partial 2-tree $G^{\prime}$. Now add the non-edges $f_{1}, f_{2}$ to give a 2-tree $H$ with a DR-plan of size 2. The Cayley configuration space $\Phi_{f}\left(G^{\prime}, \delta_{E \backslash e}\right)$ is a single interval of attainable length values $\lambda_{F}$ for the edge $f$. When $\delta$ is generic, i.e. does not admit collinearities or coincidences in the realizations of $(G, \delta)$, the realization space of $\left(H,\left\langle\delta_{E \backslash e}, \lambda_{f}\right\rangle\right)$ has 16 solutions $q_{\lambda_{f}}^{p}$ (modulo orientation preserving isometries), with distinct orientation types $\sigma_{p}$ (two orientation choices for each of the 4 triangles) that can be obtained by solving a sequence of 4 single quadratics in 1 variable (DR-plan of size 2). By subdivided binary search in the interval $\lambda_{f} \in \Phi_{f}\left(G^{\prime}, \delta_{E \backslash e}\right)$, the desired solution $p$ of $(G, \delta)$ is found when the length of the nonedge $e$ in the realization $q_{\lambda_{f}}^{p}$ is $\delta_{e}$.

In fact, we can show that $G=K_{3,3}$ is in $O M D_{1}$ by removing a single edge to reduce (as shown in Figure 6) to a tree-decomposable graph of low Cayley complexity (which includes the class of partial 2-trees). In the next Section 4.4. we discuss this issue of why the largest class of reduced graphs is desirable.

\subsection{Optimized Modification by Enlarging the Class of Reduced Graphs}

It is possible in principle to decrease $k$ for a $\mathrm{OMD}_{k}$ graph (i.e. the number of edges to be removed to ensure an isostatic completion that is decomposable with a small DR-plan) by considering reduced graphs $G^{\prime}$ (and modified graphs $H$ ) that come from a larger class than partial 2-trees but nevertheless have convex Cayley configuration spaces at least when the realization space is restricted to a sufficiently comprehensive 
orientation type. In particular, the so-called tree-decomposable graphs of low Cayley complexity [13, 14] include the partial 2-trees and many others that are not partial 2-trees. See an illustration in Figure 6 . These too result in DR-plans of size 2 or 3 , putting $G$ in the class $\mathrm{OMD}_{k}$ and thus meeting Criteria (a) and (b). The Criterion (c) is met-for example when $k=1$-because 1-dof Cayley configuration spaces of linkages based on such graphs $G^{\prime}$ are known to be single intervals when a comprehensive orientation type $\sigma_{p}$ of the sought solution $p$ is given. In addition, the bounds of these intervals are of low algebraic complexity. More precisely, the bounds can themselves be computed using a DR-plan of size 2 or 3 , i.e. the computation of these bounds is tree-decomposable. Alternatively, the bounds are in a simple quadratic or radically solvable extension field over the rationals, or they can be computed by solving a triangularized system of quadratics.

\subsection{Problem Relationships}

In this section we provide a unified view of the various problems studied in the previous 2 sections, along with formal reductions between them. We discuss their relationship to other known problems and results as well as open questions.

\subsubsection{Special Classes of Small DR-Plans}

As seen in the previous section, 2-trees and tree-decomposable graphs have not only small, but also special DR-plans that permit easy solving - essentially by solving a single quadratic at a time.

The restricted optimal DR-planning problem requires DR-plans of one of these types, which reduces to recognizing if the input graph is a 2-tree or a tree-decomposable graph for which simple near-linear time algorithms are available [55, 45] and the DR-plan is a by-product output of the recognition algorithm.

In the recombination setting, the corresponding restricted $O M D_{k}$ problem requires the reduced graph $G^{\prime}$ and its isostatic completion $H$ to be 2-trees as in Section 4.3 or to be a low Cayley complexity treedecomposable graph as in Section 4.4. Clearly these problems have deterministic polynomial time algorithms in $n$, but the algorithms run in time exponential in $k$.

We discuss the complexity of the restricted OMD problem (when $k$ is part of the input) in the openproblem Section 7.1

\subsubsection{Optimal Modification, Completion and Recombination: Previous Work and Formal Connections}

The OMD problem is closely related to a well-studied problem of completion of an underconstrained system to an isostatic one with a small DR-plan.

Observation 14. The (decision version of) the optimal completion problem (OC) from [1, [18, 56] is $O M D_{0}$.

In fact, a restricted $O C$ problem was studied by [18] requiring the completion to be tree-decomposable.

We now connect the OMD problem to the informal optimal recombination (OR) problem mentioned as motivation at the beginning of Section 4.

In order to connect the OR problem to OMD, when the input graph is the isostatic graph at the DR-plan root, we do not consider the case where the two child solved subgraphs (corresponding to already solved subsystems) have a nontrivial intersection (in this case the recombination is trivial). We only consider the case where no two child solved subgraphs (resp. two root subgraphs when the input graph is underconstrained) share more than 1 vertex. We replace such solved subgraphs by isostatic graphs as follows. If a solved subgraph shares at most one vertex with the remainder of the graph, simply replace it by an edge one of whose endpoints is the shared vertex. Otherwise, replace it by a 2-tree graph of the shared vertices. Finally, we add the additional restriction to the OM problem that when any edge in a solved subgraph is chosen among the $k$ edges to be removed, in fact the entire solved subgraph must be removed and all of its edges must be counted in $k$.

This reduction is used also for adapting algorithms for optimal DR-planning, recombination, completion, OMD, and other problems from bar-joint systems to so-called body-hyperpin, defined in Section 5 , by showing that the problems for the latter are reduced to the corresponding problems on bar-joint systems. 

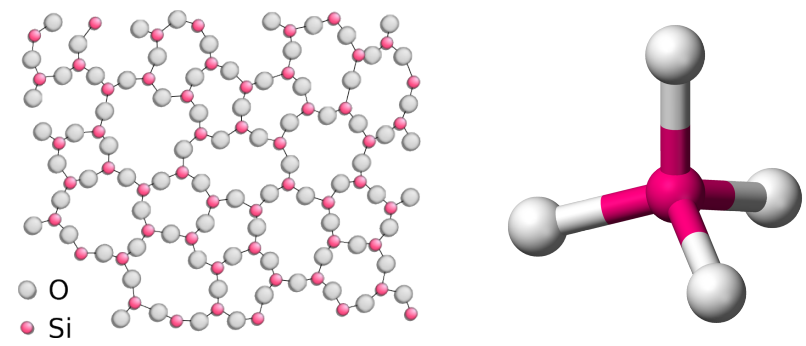

Figure 7: Example of a single monolayer of a silica (silicon and oxygen) glassy structure. This can be viewed as a triangle-multipin qusecs where the silicon atoms are the triangles and the oxygen atoms are the pins. Not shown here are other monolayers stacked on this one. Each silicon atom has the structure seen to the right, and so binds to another oxygen atom in an adjacent monolayer. Pictures taken from [57] and [58].

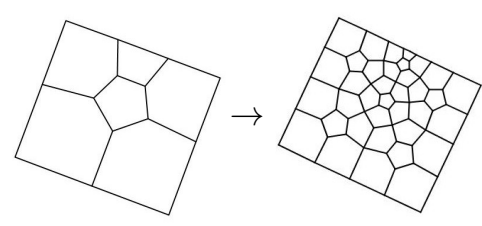

(a)

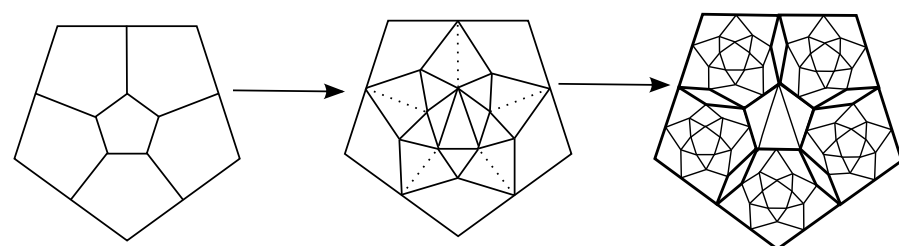

(b)

Figure 8: Examples of self-similarity via repeated subdivision. In $8 \mathrm{a}$ [59, there is a simple subdivision scheme that does not guarantee isostaticity. In (8b), a more complicated scheme is used, ensuring that the resulting graph is isostatic but not tree-decomposable.

\section{Application: Finding Completions of Underconstrained Glassy Structures from Undercon- strained to Isostatic}

We can use qusecs DR-plans to design materials such as disordered graphene and silica bi-layers 31. 32. We investigate a more specific problem in a somewhat more general setting: the problem of finding boundary conditions (additional constraints) to add to an underconstrained monolayer to make it isostatic. This can be done in a number of ways: (1) pin together 2 underconstrained monolayers in such a way that the resulting bi-layer becomes isostatic (see Figure 7); (2) pin the boundary of (or in general, add constraints to) a layer (possibly a genus 0 monolayer) so that it becomes isostatic; or (3) design a broader class of structures to ensure they are isostatic, self-similar (via some subdivision rule) and in addition isostatic at each level of the subdivision (see Figure 8p.

In all cases, we are specifically interested in how to add additional constraints such that the resulting isostatic structure has a small DR-plan; this way a realization can be found, allowing efficient stress, flex and other property design related to the rigidity matrix. To answer these questions, we first introduce the qusecs' that are used to model these materials. In this section, we discuss Item (2) in detail.

\subsection{Body-Hyperpin Qusecs}

Definition 15. A body-hyperpin qusecs is a constraint system where the objects are rigid bodies, subsets of which are pinned together by pins; i.e, are incident at a common point.

Remark* 16. A body-hyperpin qusecs is a special case of bar-joint qusecs of the previous sections of the paper. As such, the DR-planning for isostatic systems discussed in Section 3 is unchanged and the results of Section 4 still go through with minor modifications.

For the remainder of this section, we deal only with the DR-plan of such qusecs. Hence, we refer only to the combinatorics or underlying hypergraph of the qusecs. We now introduce 2 sub-classes of body-hyperpin 
graphs for modeling Examples 4 and 5 in Section 1, for which the optimal completion problem is significantly easier.

Definition 17. A body-pin graph is a body-hyperpin graph with the following conditions: (1) each pin is shared by at most two bodies; and (2) no two bodies share more than one pin

Such a body-pin graph, $G_{B P}$, can also be seen as a body-bar graph, $G_{B B}$, where the bodies of $G_{B B}$ are the original bodies of $G_{B P}$ and each pin between bodies in $G_{B P}$ are replaced with 2 bars in $G_{B B}$ between the same bodies. Such body-bar graphs with 1 and 2-dof can be characterized by being $(3,4)$ and $(3,5)$-tight respectively [43] [5] (defined in 2.1). See Figure 9b.

Definition 18. A triangle-hyperpin graph is a body-hyperpin graph where each body is a triangle, i.e., it shares pins with at most 3 other bodies. This is also represented as a hyper-graph where each pin is a vertex and each triangle represents a tri-hyperedge. For such hypergraphs, 1 and 2-dof can be characterized by $(2,4)$ and $(2,5)$-tightness respectively [43] [5].

Body-pin graphs are of particular interest to us in the context of Example 4 in Section 1. Trianglemultipin graphs can be used to represent the silica bi-layers and glassy structures described in Example 5 of Section 1, where each triangle is the junction of "disks" in the plane (see Figure 7). Typically, these systems are not isostatic, so to relate the work of this paper to the systems, we define a slightly different kind of DR-plan using the notion of $(k, l)$-sparsity and tightness.

Definition 19. A $(k, l)$-tight DR-plan is one in which each child node is either a vertex maximal proper $(k, l)$-tight sub-graph of the parent node or it is trivial. In our case, the trivial nodes are the bodies.

Provided such $(k, l)$-sparse graphs are matroidal (conditions given in [43]), the notion of a canonical DR-plan extends directly to the case when the hypergraph is $(k, l)$-sparse (i.e., independent) using the straightforward notion of trivial and non-trivial intersections and $(k, l)$-tightness conditions as in Section 3. In particular, we define canonical DR-plans with similar properties for the 1 and 2-dof body-pin and triangle-hyperpin systems defined above.

Observation* 20. For the 1-dof body-pin graphs described above that are $(3,4)$-sparse, a $(3,4)$-tight canonical DR-plan exists where every node of a $(3,4)$-sparse graph satisfies one of the following: (1) its children are 2 proper vertex-maximal 1-dof graphs that intersect on another 1-dof graph; or (2) its children are all of the proper maximal 1-dof sub-graphs, pairwise sharing at most one body.

As in Section 3, a strong Church-Rosser property holds, making all canonical DR-plans optimal:

Observation* 21. When the input is independent, all $(3,4)$-tight canonical DR-plans are optimal. We can find such a DR-plan in the same time complexity as the (2,3)-tight case for bar-joint graphs discussed in Section 3

The above-mentioned algorithm exists because such $(3,4)$-tight graphs are matroidal and have a pebble game [43].

The above discussion leads to the main theorem:

Theorem* 22. Given a 1-dof body-pin or triangle-multipin graph and corresponding 1-dof DR-plan, there is a quadratic algorithm for the 1-dof optimal completion problem of Section 4.

Observation* 23. For the 2-dof case, provided an analogous statement to Observation 21 holds, then Theorem 22 holds for the 2-dof systems.

Remark 24. While the proof for Theorem 22 gives us a DR-plan for the isostatic completion with minimum fan-in, (a reasonable measure of algebraic complexity), a more nuanced measure that treats solutions of 1-dof and 2-dof systems as 1 or 2 parameter families would no longer be optimized by the algorithm given in that proof. In particular, the complexity of the standard algorithm in the $k$-dof case would be exponential in $k$ (even if the case were matroidal and an optimal DR-plan is known). 


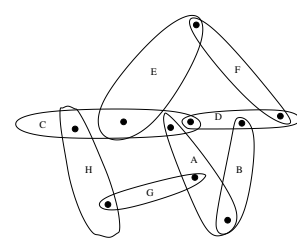

(a)

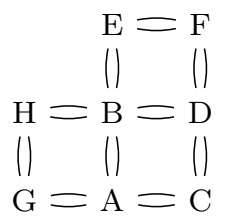

(b)

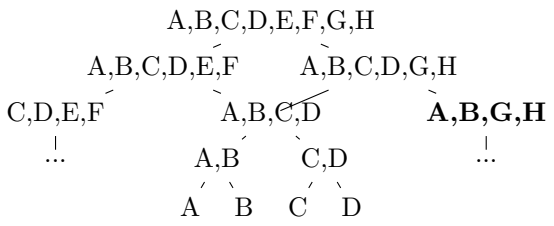

(c)

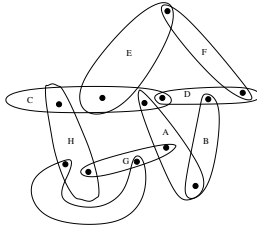

(d)

Figure 9: 9a A 1-dof body-pin graph. 9b The corresponding body-bar graph, explained in Definition 17 (9c) The 1-dof DR-plan for the graph. In this case, to obtain an isostatic system, we would need to add a body and 2 pins to one of the nodes in the second level. (9d) The result of adding one such body to the bold-faced node.

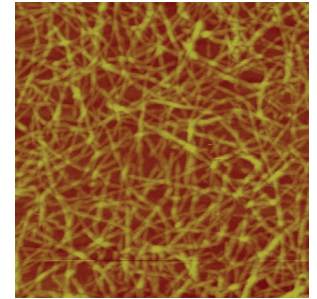

(a)

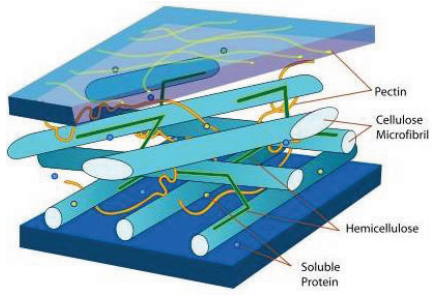

(b)

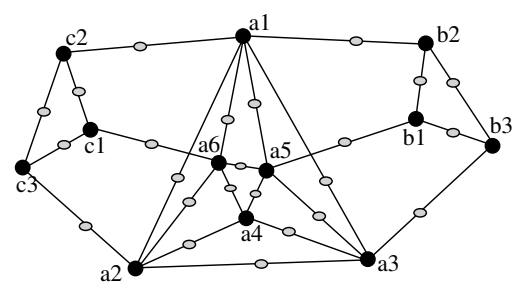

(c)

Figure 10: 10a Microfibrils of carboxymethylated nanocellulose adsorbed on a silica surface 25. 10b. Cross-linking of cellulose microfibrils [26]. 10c An isostatic pinned line-incidence graph, representing fibrils and their attachments.

\section{Application: Finding Optimal DR-Plans and Realizations for Cross-Linking Microfibrils}

The canonical DR-plan of Section 3 can additionally be applied to analyze and solve the structure of cross-linking collagen microfibrils in animals, cellulose microfibrils in plant cell walls, and actin filaments in the cytoskeleton by modeling these structures as a third type of qusecs, pinned line-incidence systems.

Collagen is an important protein material in biological tissues with highly elastic mechanical properties [60. Cellulose is the most important constituent of the cell wall of plants (see Figure 10a) 61, 62. Both of these substances consist of a large number microfibrils, each of which is cross-linked at 2 places with usually 3 other fibrils, where the cross-linking is like an incidence constraint that the crosslinked fibrils can slide against each other while remaining incident (see Figure 10b.

\subsection{Modeling the Fibrils as a Pinned Line-Incidence System}

The cross-linking microfibrils can be modeled as a pinned line-incidence constraint system in $\mathbb{R}^{2}$, where incidence constraints are used instead of distance constraints.

A pinned line-incidence system $(G, \delta)$ is a graph $G=(V, E)$ together with parameters $\delta$ specifying $|E|$ pins with fixed positions in $\mathbb{R}^{2}$, such that each edge is constrained to lie on a line passing through the corresponding pin, i.e. $\delta: E \rightarrow \mathbb{R}^{2}$. A pinned line-incidence graph $G$ is rigid if $|E|=2|V|$ and $\left|E^{\prime}\right| \leq 2\left|V^{\prime}\right|$ for every induced subgraph $\left(V^{\prime}, E^{\prime}\right)$ [36]. Note that no trivial motion exists since the pins have fixed positions on the plane. Euclidean transformations are not factored out. In particular, both a single vertex and a single edge are underconstrained graphs.

In the case of microfibril cross-linking, each fibril is attached to some fixed larger organelle/membrane at one site. Consequently, each fibril can be modeled as an edge of the graph, with the attachment being the corresponding pin. The two cross-linkings in which the fibril participates are modeled as the two vertices in $V$ defining the edge. 
Figure 10c shows an example of a pinned line-incidence graph, where the grey ovals denote pins representing attachments of fibrils, and the vertices $a_{1}, a_{2}, \ldots, c_{3}$ represent cross-linkings. The graph is isostatic, with 12 vertices and 24 edges/pins.

\subsection{Optimal DR-Plan for Pinned Line-Incidence Systems}

In this section, we will adapt the results in Section 3 to give the canonical DR-plan for pinned lineincidence graphs. First, we note that an isostatic pinned line-incidence graph can be disconnected, being the disjoint union of two or more isostatic subgraphs. This is because the pins have fixed positions on the plane. We define a trivial graph to be a single vertex and make the following modification to the definition of the canonical DR-plan:

Definition 25. The DR-plan of a pinned line-incidence graph $G$ is one in which (1) each child node of a non-leaf node $C$ is either a connected rigid vertex-induced subgraph of $C$, or an edge not contained in any proper rigid subgraph of $C$, and (2) a leaf node is a single edge.

The canonical DR-plan of $G$ is one in which the child rigid subgraphs are connected, isostatic vertexmaximal subgraphs of the parent.

Theorem 4 holds for pinned line-incidence graphs with this modified definition. The proof is similar to the original proof (in Section 3) using the same set of lemmas and the following modified version of Observation 5, which can be proved using a simple counting based argument.

Observation 26. Let $F_{i}$ and $F_{j}$ to be subgraphs of the same isostatic graph $F$, where each of them can be either a single edge or a connected isostatic subgraph. There are only two possible cases: (1) at least one of $F_{i}, F_{j}$ is an edge, if and only if $F_{i} \cup F_{j}$ is underconstrained, if and only if $F_{i} \cap F_{j}$ is trivial; and (2) both $F_{i}$ and $F_{j}$ are isostatic, if and only if $F_{i} \cup F_{j}$ is isostatic, if and only if $F_{i} \cap F_{j}$ is isostatic.

Given Observation 26, Lemma 6. Point 1 and 3, straightforwardly extend to pinned line-incidence graphs. The proof of Point 2 for pinned line-incidence graphs is given in Appendix A.4.1. Thus it is straightforward to adapt the proof of Theorem 4 to pinned line-incidence graphs. Consequently, we can efficiently find the optimal DR-plan for pinned line-incidence graphs using basically the same algorithm as for bar-joint graphs.

Note: The recombination problem for pinned line-incidence systems is trivial. Since the pins are given fixed positions in the plane, the solutions of a isostatic sub-system will automatically be consistent with solutions of the remaining of the system.

\section{Conclusion}

We have clarified the main source of complexity for the optimal DR-plan and recombination problems. For the former problem, when there are no overconstraints (as is the case for $2 \mathrm{D}$ qusecs whose realizations are many common types of layered materials), we defined a canonical DR-plan and showed that any canonical DR-plan is guaranteed to be optimal, a strong Church-Rosser property. This gives an efficient $\left(O\left(n^{3}\right)\right)$ algorithm to find an optimal DR-plan that satisfies other desirable characteristics.

We have also described a novel method of efficiently realizing a $2 \mathrm{D}$ qusecs from the optimal DR-plan by modifying the otherwise indecomposable systems at nodes of a DR-plan. These results rely on a recent theory of convex Cayley configuration spaces. Relationships and reductions between these and previously studied problems were formally clarified.

We then modeled specific layered materials using extensions of the above theoretical results including the motivating Examples 1-5 in the introduction. 


\subsection{Open Problems}

The first set of problems are from Section 3

Open Problem 1. Is there a more efficient algorithm than $O\left(|V|^{3}\right)$ to find the canonical DR-plan of isostatic 2D bar-joint graphs?

Conjecture 27. The Modified Frontier Algorithm (MFA) [8] finds a canonical, and hence optimal, DR-plan.

The difficulty of proving Conjecture 27 arises from the fact that MFA, although running in time $O\left(n^{3}\right)$, is a bottom-up algorithm, involving complex datastructures. However, a proof of optimality, even if it exists, would not be possible without the new notion of a canonical DR-plan at hand. The intuition for this conjecture comes from the similarity of the DR-plan generated by MFA to that of the sequential decomposition described in the proof of Theorem 9 . Since it is known 8 that the DR-plan generated by MFA is cluster-minimal, an alternate conjecture is the following.

Conjecture 28. For independent graphs, cluster-minimal DR-plans are optimal. In fact, for independent graphs, cluster-minimality and canonical are equivalent properties of a DR-plan.

Open Problem 2. Although generic rigidity is a property of graphs, and moreover, in the case of qusecs, generic rigidity has a combinatorial sparsity and tightness-based characterization, the original definition of independence in the rigidity matroid requires an algebraic notion of independence of vectors of indeterminates over $\mathbb{R}$. Thus the definition of the DR-plan requires algebra over the reals. In fact, the recursive decomposition problem is not tied to geometric constraint graphs or an algebraic-geometric or mechanical notion of rigidity, and can be defined for any graph using the notion of an abstract rigidity matroid 63 . This is a type of matroid with two additional matroid axioms; abstract rigidity matroids can be defined in a purely graph-theoretic manner, with no need for algebra in their definition. However, such abstract rigidity need not have a sparsity characterization. On the other hand, there are sparsity matroids that do not correspond to any notion of abstract rigidity. However, when an abstract rigidity matroid is also a sparsity matroid, then the techniques of this paper directly apply and we can obtain purely combinatorially defined recursive decompositions of graphs.

A few natural open questions concern the following common theme that runs through the optimal recombination and later sections of the paper:

Open Problem 3. For fixed $k$, we have polynomial time optimal DR-planning (Section 3), recombination (modification) in the presence of $k$ overconstraints, optimal modification for decomposition $\operatorname{OMD}_{k}(G)$ when at most $k$ constraints are removed (Section 4 ), and also optimal completion using at most $k \leq 2$ constraints in the body-pin and triangle-multipin cases for a somewhat different optimization of the DR-plan (Section 4.5). However, in the running time of all of these algorithms, $k$ appears in the exponent. Can $k$ be removed from the exponent?

One problem in the above theme is from Section 5.

Open Problem 4. What is the complexity of the optimal completion problem when the given graph has more than 2-dofs? Our proof for the 1 and 2-dof cases relied heavily on the matroidal properties of their corresponding $(k, l)$-tightness. For higher number of dofs, the $(k, l)$ characterization is no longer matroidal 43. As a result, the major obstacle is that there is no easy way of obtaining an optimal or canonical $k$-dof DR-plan in general. Even assuming such a DR-plan is available, if higher dofs had the same characteristics, Observation 24 raises questions about the correct measure of DR-plan size that captures algebraic complexity for recombining graphs with many dofs (this is not an issue in the isostatic case). Unless some restrictions can be found and taken advantage of, the $k$-dof optimal completion problem would have complexity exponential in $k$.

Another problem from the above theme is from Section 4 
Open Problem 5. What is the complexity of the restricted OMD (optimal modification for decomposition) problem? This has the potential to be difficult. For example, when the isostatic completion is required to be a 2-tree the restricted OMD problem is reducible to the maximum spanning series-parallel subgraph problem shown by 64 to be NP-complete even if the input graph is planar of maximum degree at most 6. However, since the OMD problem has other input restrictions such as not having any proper isostatic subgraphs, it is not clear if the reverse reduction exists and hence it is unclear whether the OMD problem is NP-complete.

The same holds for the restricted OMD problem where the isostatic completion is required to be a treedecomposable graph of low Cayley complexity (i.e. have special, small DR-plans). One potential obstacle to an indecomposable graph $G$ 's membership in the restricted $\mathrm{OMD}_{k}$ for small $k$ is if $G$ is tri-connected and has large girth. In fact, 6-connected (hence rigid) graphs with arbitrarily large girth have been constructed in 65 .

The next is the reverse direction of Observation 14 in Section 4.5

Open Problem 6. Is the OMD (optimal modification for decomposition) problem reducible to the OC (optimal completion) problem?

More general problem directions are the following.

Open Problem 7. Combinatorial rigidity for periodic structures is an active area of research. This paper motivates a study of the rigidity of self-similar structures, with self-similar groups replacing periodic groups.

Open Problem 8. The pinned line incidence structures of Section 6, for example in the case of collagen microfibrils, whose function is elastic contraction, should be considered congruent under projective transformations. I.e. the projective group should be factored out as a trivial motion in a new project for extending the combinatorial rigidity characterization of such systems. (Currently we permit no trivial motions at all).

Open Problem 9. Experimental validation (either computationally or physical experiments) of predications based on the material model and theory used in this paper. This can be done by modeling known materials and putting stresses on them, seeing if the prediction is observed in the real material. Or, our theory could be used to design new materials, which can be tested to see if they possess predicted properties.

\section{Appendix A. Proofs}

\section{Appendix A.1. Proofs from Section 3}

Appendix A.1.1. Proof of Observation 5

Proof. For (1), simply note that if $F_{i} \cup F_{j}$ were trivial, then, by definition, $F_{i}$ and $F_{j}$ must be trivial.

For the next parts, we use the quantity $d(G)=2|V|-|E|$, which we call density. For (2), observe that underconstrained subgraphs of isostatic graphs must have density less than 3. For (3), observe that, given an isostatic graph, a subgraph with density 3 must also be isostatic. Then, use the fact that, by definition, $d\left(F_{i}\right)=3$ and $d\left(F_{j}\right)=3$. Then it is straightforward application of the inclusion-exclusion $d\left(F_{i} \cap F_{j}\right)=d\left(F_{i}\right)+d\left(F_{j}\right)-d\left(F_{i} \cup F_{j}\right)$.

For (4), because subgraphs of a isostatic graph can only be trivial, underconstrained, or isostatic, all cases have already been exhausted.

\section{Appendix A.1.2. Proof of Lemma 6, Point 1}

Proof. Assume $C_{i} \cup C_{j} \neq C$. This would contradict the proper vertex-maximality of $C_{i}, C_{j}$. In the reverse direction, we know $C$ is either a non-leaf node (isostatic by definition of a DR-plan) or $G$ itself (isostatic by definition of the problem). Thus, $C_{i} \cup C_{j}=C$ is isostatic. 
Appendix A.1.3. Proof of Lemma 6, Point 2

Observation 29. Take $R_{i}=C \backslash C_{i}$ and $R_{j}=C \backslash C_{j}$. If $C_{i} \cup C_{j}$ is isostatic, then there can be no edges in $C$ between the vertices of $R_{i}$ and $R_{j}$.

Proof. Lemma 6, Point 1, shows that $C_{i} \cup C_{j}$ must equal the parent graph $C$.

Proof. (Of Lemma 6, Point 2)

The alternative phrasing is as in Lemma 6, Point 1 .

Take $R_{i}=C \backslash C_{i}, R_{j}=C \backslash C_{j}$, and $D_{i, j}=C_{i} \cap C_{j}=\left(C \backslash R_{i}\right) \backslash R_{j}$ (note that $R_{j} \subset C_{i}, R_{i} \subset C_{j}$ and $D_{i, j} \subset C_{i}, C_{j}$ ). Furthermore, take the proper subgraphs $R_{i}^{\prime} \subset R_{i}, R_{j}^{\prime} \subset R_{j}$, and $D_{i, j}^{\prime} \subset D_{i, j}$ that are non-empty.

Assume that there is a third isostatic vertex-maximal proper subgraph $C_{k}$ (with $C_{k}^{\prime}=C \backslash C_{k}$ ). There are $3 \times 3 \times 3=27$ possible cases for what this subgraph could be.

Without loss of generality, all graphs are the induced subgraphs of $C$.

- 3 cases: $C_{k}$ cannot be $C=R_{i} \cup R_{j} \cup D_{i, j}, C_{i}=R_{j} \cup D_{i, j}$, or $C_{j}=R_{i} \cup D_{i, j}$. This is by definition.

- 13 cases: $C_{k}$ cannot be a proper subgraph of $C_{i}$ and $C_{j}$ or else $C_{k}$ would not be vertex-maximal. These are the graphs $R_{i}^{\prime} \cup D_{i, j}, R_{j}^{\prime} \cup D_{i, j}, D_{i, j}, R_{i} \cup D_{i, j}^{\prime}, R_{j} \cup D_{i, j}^{\prime}, R_{i}^{\prime} \cup D_{i, j}^{\prime}, R_{j}^{\prime} \cup D_{i, j}^{\prime}, D_{i, j}^{\prime}, R_{i}, R_{j}$, $R_{i}^{\prime}, R_{j}^{\prime}$, and $\emptyset$.

- 2 cases: $C_{k}$ cannot contain $C_{i}$ or $C_{j}$ as proper subgraphs, or else they are not vertex-maximal. These are the graphs $R_{i}^{\prime} \cup R_{j} \cup D_{i, j}$ and $R_{i} \cup R_{j}^{\prime} \cup D_{i, j}$ respectively.

- 4 cases: $C_{k}$ cannot be $R_{i} \cup R_{j}, R_{i}^{\prime} \cup R_{j}, R_{i} \cup R_{j}^{\prime}$, or $R_{i}^{\prime} \cup R_{j}^{\prime}$ because these are all disconnected (Observation 29) and cannot be isostatic.

- 1 case: $C_{k}=R_{i}^{\prime} \cup R_{j}^{\prime} \cup D_{i, j}$ is not possible. Since $C_{i} \cup C_{k}=R_{i}^{\prime} \cup R_{j} \cup D_{i, j} \neq C$ we have from Lemma 6, point 1 that $C_{i} \cup C_{k}$ cannot be isostatic. We also know it cannot be trivial because it contains isostatic subgraphs. This means it must be underconstrained. From Observation 5 , we know that $C_{i} \cap C_{k}=R_{j}^{\prime} \cup D_{i, j}$ must then be trivial. This is impossible because $D_{i, j}$ is isostatic, thereby contradicting the assumption that $C_{k}$ is isostatic.

- 1 case: $C_{k}=R_{i}^{\prime} \cup R_{j}^{\prime} \cup D_{i, j}^{\prime}$ is not possible. Since $C_{i} \cup C_{k} \neq C$ (and $C_{j} \cup C_{k} \neq C$ ), we know by the same logic as the previous case that the $C_{i} \cap C_{k}$ must be trivial (a single node). However, $C_{i} \cap C_{k}=R_{j}^{\prime} \cup D_{i, j}^{\prime}$. This causes a contradiction, the intersection cannot be trivial because $R_{j}^{\prime}$ and $D_{i, j}^{\prime}$ are not empty sets and are disjoint.

- 2 cases: $C_{k}=R_{i}^{\prime} \cup R_{j} \cup D_{i, j}^{\prime}$ and $C_{k}=R_{i} \cup R_{j}^{\prime} \cup D_{i, j}^{\prime}$ are not possible. The proof mirrors the previous case, except here you must choose $C_{i}$ and $C_{j}$ respectively.

- 1 case: $C_{k}=R_{i} \cup R_{j} \cup D_{i, j}^{\prime}$ is all that remains.

Since $D_{i, j} \subset C_{i}, C_{j}$ it means that $C_{k} \cup C_{i}=C_{k} \cup C_{j}=C$, thus proving the Lemma.

Appendix A.1.4. Proof of Lemma 6, Point 3

Proof. Assume there is some $k$ such that $C_{i} \cap C_{k}$ is not trivial. By Observation 5 , $C_{i} \cap C_{k}$ must be isostatic. Then, by Lemma 6 point 2, the intersection between any two children must be isostatic. This means that $C_{i} \cap C_{j}$ is isostatic. Therefore, such a $k$ cannot exist and all intersections are trivial. 


\section{Appendix A.2. Proofs from Section 4}

Appendix A.2.1. Proof of Theorem 12

Proof. The Cartesian realization space of $\left(H,\left\langle\delta_{E^{\prime}}, \lambda_{F}\right\rangle\right)$ is computed easily with a DR-plan of size 2, and is the union of $2^{t}$ solutions (modulo orientation preserving isometries) each with a distinct orientation type, where $t$ is the number of triangles in the 2-tree $H$; here $\delta_{S}$ is the restriction of the length vector $\delta$ to the edges in $S$. A desired solution $p$ (or connected component of a solution space) of $(G, \delta)$ of an orientation type $\sigma_{p}$ can be found by a subdivided binary search of the Cartesian realization space of $\left(H,\left\langle\delta_{E^{\prime}}, \lambda_{F}\right\rangle\right)$ of orientation type $\sigma_{p}$, as $\lambda_{F}$ ranges over the discretized convex polytope $\Phi_{F}\left(G^{\prime}, \delta_{E}^{\prime}\right)$ with bounding hyperplanes described in Theorem 10. A solution $p$ is found when the lengths for nonedges in $D$ match $\delta_{D}$.

\section{Appendix A.3. Proofs from Section 5}

Appendix A.3.1. Proof of Remark 16

Proof. We can replace each body that has only one pin by a single vertex. A body with 2 pins can be replaced by an edge. In general, a body with $n$ pins can be replaced by a 2 -tree on $n$ vertices. When finding a DR-plan, we treat each body as trivial, so they become the leaves of the DR-plan. The optimal recombination problem and approach of Section 4 are unchanged. The optimal completion via the optimal modification problem in Section 4 now has an additional restriction that all edges in the 2-tree representation of the bodies must be removed together, not individually.

\section{Appendix A.3.2. Proof of Observation 20}

Proof. The existence of this canonical DR-plan follows from the same arguments as in the proof of Theorem 4. The only difference is the definition of a trivial intersection. In this case, when two subgraphs share more than 1 body, they become rigid (in fact over constrained). Sharing a pin is not considered an intersection. Such a structure is viewed as two subgraphs each sharing 1 body with a third 1-dof subgraph which essentially just consists of those two bodies pinned together.

\section{Appendix A.3.3. Proof of Theorem 22}

Proof. Suppose we are given a body-pin graph and its corresponding body-bar graph $G$ and have obtained the 1-dof DR-plan $T$. Each node of $T$ is then a vertex-maximal proper 1-dof subgraph of $G$.

To make the graph isostatic, we need only add one body and pin it to 2 other bodies. Doing so will cause $G$ to become (3,3)-tight.

We adopt the following algorithm. Choose the 2 bodies to pin to by choosing a node $b$ in $T$ and looking at its children. From Observation 21, we know that the children can only share a single pin or a sub-graph. Pin the new body to bodies in two separate children. Doing so will ensure that all children of $b$ will have 1-dof and all ancestors of $b$ (including $b$ ) will now be isostatic.

Such a pinning covers all possible ways of adding a new body. Assume a new body $b$ is added to the input graph and pin it to $b_{i}$ and $b_{j}$ to make it isostatic. Then, there is a lowest 1-dof node $v$ in $T$ such that $b_{i}$ and $b_{j}$ appear in $v$. Thus, pinning $v$ in the manner described yields an equivalent isostatic DR-plan to pinning $b$ to $b_{i}$ and $b_{j}$.

For each node $b$, assign a size of the $T_{b}$ denoted $\left|T_{b}\right| .\left|T_{b}\right|=\max _{v \in T_{b}} \operatorname{fanin}(v)$. We are looking for $b$ that minimizes $\left|T_{b}\right|$. Denote the sub-tree of $T$ rooted at $v$ by $T^{v}$ and the number of leaves in a tree $T$ by $n l(T)$. Note that $f a n i n(b)=n l\left(T^{b}\right)$ because no descendant of $b$ is isostatic. Similarly, for any ancestor $w$ of $b$, $\operatorname{fanin}(w)=n l\left(T^{w}\right)-n l\left(T^{b^{\prime}}\right)+1$, where $b^{\prime}$ is the child leading to $b$. All other nodes are not isostatic and hence do not appear in the isostatic DR-plan.

The node to be pinned is always the the deepest nontrivial node of some path in $T$. Suppose a node $b$ is pinned that has a nontrivial child $v$. Then, $\operatorname{fanin}_{b}(b)=n l\left(T^{b}\right)=n l\left(T^{v}\right)+n$, where $n$ is essentially the number of leaves between $b$ and $v$. If we had instead chosen to pin $v$, then $\operatorname{fanin}_{v}(b)=n l\left(T^{b}\right)-n l\left(T^{b^{\prime}}\right)+1 \leq$ $\operatorname{fanin}_{b}(v)$. And for each ancestor $w$ of $b, \operatorname{fanin}(w)$ is unchanged, meaning $\left|T_{v}\right| \leq\left|T_{b}\right|$. Thus we only have to check the deepest non-trivial nodes.

Running the above algorithm brute force gives running time quadratic in the number of bodies of the given body-pin system. 
For the multi-triangle pin graphs, we can do the same thing, except we need to add a single triangle to one of the nodes to cause it to become isostatic.

\section{Appendix A.3.4. Proof of Observation 23}

Proof. The only difference from the 1-dof case is that now we need to remove 2-dofs from our graph. Start with a 2-dof DR-plan T. Like in the previous proof, we need to add a body and 2 pins to 2 nodes to obtain an isostatic DR-plan.

Suppose we pin 2 distinct nodes $v_{i}$ and $v_{j}$. Then, there must exist a common ancestor $a$ of $v_{i}$ and $v_{j}$. Then, in $T_{v_{i}, v_{j}}$, fanin $_{v_{i}, v_{j}}(a)=n l\left(T^{a}\right)$. However, if we chose to pin one of $v_{i}$ and $v_{j}$ twice, then $\operatorname{fanin}_{v}(a)=n l\left(T^{a}\right)-n l\left(T^{a^{\prime}}\right)+1$. Thus $\operatorname{fanin}_{v}(a)^{\prime} \leq \operatorname{fanin}_{v_{i}, v_{j}}(a)$. All ancestors of $a$ are unchanged. So $\left|T_{v}\right| \leq\left|T_{v_{i}, v_{j}}\right|$.

Thus the only choice is to pin a single node twice. Hence, we can run the same algorithm as the 1-dof case and simply pin twice instead of once.

\section{Appendix A.3.5. Proof of Observation 24}

Proof. An isostatic graph has 3 parameters that define its position and orientation. These are the Euclidean motions. A 1-dof graph has 4 parameters: the 3 Euclidean motions and a dof parameter. A 2-dof has 5 parameters. The number of parameters roughly correlates with the algebraic complexity of obtaining a realization.

Thus, starting with a $T$ as described in the proof for Remark 22 , when a node $b$ is pinned, the same structure is preserved as before. Suppose $v$ is an isostatic node after pinning $b$. Then, the children of $v$ (except one if $v \neq b$ ) have 1-dof. The realization complexity for $v$ is simply that of realizing each of its children. In general, the number of parameters for $v$ will be $n p(v)=4 n c_{1}(v)+3$, if $v \neq b$ and $n p(b)=4 n c_{1}(b)$, where $n c_{k}(v)$ is the number of $k$-dof children of $v$.

Minimizing the algebraic complexity requires minimizing the maximum $n p(v)$ for any node $v$. In this case, it is not possible to always choose to pin a node closest to a leaf in the tree, because it could have high fan-in. So we try brute force by pinning all nodes to pick the one with the lowest algebraic complexity. This algorithm is still quadratic for the 1-dof case.

For the 2-dof situation, there are more cases to consider. If we pin the same node twice as above, we have $n p(v)=5 n c_{2}(v)+3$ for any ancestor $v \neq b$ and $n p(b)=5 n c_{2}(b)$. If we pin a node $v$ and one of its ancestors $v^{\prime}$, then any nodes between $v^{\prime}$ and $v$ will be 1-dof, any nodes above $v^{\prime}$ will be isostatic, and nodes below $v$ will be 2 -dof. Note that solving or realizing $v^{\prime}$ will also realize $v$. Next, we need to consider nodes above and including $v^{\prime}$ in our complexity: $n p\left(v^{\prime}\right)=5 n c_{2}\left(v^{\prime}\right)+4$ and $n p(a)=3+5 n c_{2}(a)$ for $a$ an ancestor of $v^{\prime}$.

The only remaining case is pinning two nodes that are incomparable, i.e. do not have a descendant/ancestor relationship. The only change from the previous case is that for the lowest common ancestor of the nodes $v^{\prime}, n p\left(v^{\prime}\right)=2 * 4+5 n c_{2}\left(v^{\prime}\right)$. For any ancestor $a$ of $v^{\prime}$, we still have $n p(a)=3+5 n c_{2}(a)$.

Like the 1-dof case, we again cannot simply choose the nodes deepest in the tree to pin. However, neither can we assume pinning one node twice will give us the best algebraic complexity. Hence, we will need to check each pair of nodes to pin. This makes our brute-force algorithm $O\left(b^{3}\right)$, where $b$ is the number of bodies.

\section{Appendix A.4. Proofs from Section 6}

Appendix A.4.1. Proof of Lemma 6, Point 2 - for 2-dimensional pinned line incidence graphs

Proof. We use the same notation as in the original proof of Lemma 6, Point 2, given above. Without loss of generality, all graphs are the induced graphs on $C$.

First notice that since $C_{i} \cup C_{j}$ is isostatic, by Observation 26, both $C_{i}$ and $C_{j}$ are connected isostatic vertex-maximal proper subgraphs of $C$. Since $C_{j} \cup C_{j}=C$, there are no edges in $C$ that is not contained in a isostatic subgraph, so $C$ does not have any single-edge child node, and $C_{k}$ is a connected isostatic vertex-maximal proper subgraph of $C$.

We analyze all the possible cases for $C_{k}$. 
- 1 case: $C_{k}=R_{i}^{\prime} \cup R_{j}^{\prime} \cup D_{i, j}$ is not possible. Since $C_{k} \cup C_{i}=R_{i}^{\prime} \cup R_{j} \cup D_{i, j} \neq C$ we have from Lemma 6. Point 1, that $C_{k} \cup C_{i}$ cannot be isostatic. By Lemma 26 it must be underconstrained, so one of $C_{i}$ and $C_{k}$ must be an edge, contradicting the assumption that both $C_{i}$ and $C_{k}$ are isostatic.

- 1 case: $C_{k}=R_{i}^{\prime} \cup R_{j}^{\prime} \cup D_{i, j}^{\prime}$ is not possible. The proof is similar to the previous case.

- 2 cases: $C_{k}=R_{i}^{\prime} \cup R_{j} \cup D_{i, j}^{\prime}$ and $C_{k}=R_{i} \cup R_{j}^{\prime} \cup D_{i, j}^{\prime}$ are not possible. The proof is similar to the previous case.

All remaining cases are similar to the original proof for 2D bar-joint graphs.

\section{References}

[1] M. Sitharam, Combinatorial approaches to geometric constraint solving: Problems, progress, and directions, In: DIMACS: Series in Discrete Mathematics and Theoretical Computer Science 67 (2005) 117.

[2] C. M. Hoffman, A. Lomonosov, M. Sitharam, Decomposition plans for geometric constraint systems, part i: Performance measures for cad, Journal of Symbolic Computation 31 (4) (2001) 367-408. doi:10.1006/jsco.2000.0402

[3] C. M. Hoffman, A. Lomonosov, M. Sitharam, Decomposition plans for geometric constraint problems, part ii: new algorithms, Journal of Symbolic Computation 31 (4) (2001) 409-427. doi:10.1006/jsco.2000.0403

[4] G. Laman, On graphs and rigidity of plane skeletal structures Journal of Engineering Mathematics 4 (1970) 331-340, 10.1007/BF01534980. URL http://dx.doi.org/10.1007/BF01534980

[5] I. Streinu, L. Theran, Sparse hypergraphs and pebble game algorithms, European Journal of Combinatorics 30 (8) (2009) 1944-1964. doi:10.1016/j.ejc.2008.12.018

[6] T.-S. Tay, Rigidity problems in bar and joint frameworks, Ph.D. thesis, University of Waterloo (1976).

[7] N. White, W. Whiteley, The algebraic geometry of motions of bar-and-body frameworks, SIAM Journal on Algebraic Discrete Methods 8 (1) (1987) 1-32.

[8] A. Lomonosov, Graph and combinatorial analysis for geometric constraint graphs, Ph.D. thesis, University of Florida (2004).

[9] M. Sitharam, J. Peters, Y. Zhou, Optimized parametrization of systems of incidences between rigid bodies, Journal of Symbolic Computation 45 (4) (2010) 481-498.

[10] M. Sitharam, Well-formed systems of point incidences for resolving collections of rigid bodies, International Journal of Computational Geometry \& Applications 16 (05n06) (2006) 591-615.

[11] M. Sitharam, Y. Zhou, J. Peters, Reconciling conflicting combinatorial preprocessors for geometric constraint systems, International Journal of Computational Geometry \& Applications 20 (06) (2010) 631-651.

[12] M. Sitharam, H. Gao, Characterizing graphs with convex and connected configuration spaces, Discrete \& Computational Geometry 43 (3) (2010) 594-625. doi:10.1007/s00454-009-9160-8

[13] M. Sitharam, M. Wang, H. Gao, Cayley configuration spaces of 2d mechanisms part i: extreme points, continuous motion paths and minimal representations, Under review. arXiv preprint arXiv:1112.6008 (2011). arXiv:1112.6008

[14] M. Sitharam, M. Wang, H. Gao, Cayley configuration spaces of 2d mechanisms part ii: combinatorial characterization, Under review. arXiv preprint arXiv:1112.6009 (2011). arXiv:1112.6009

[15] M. Sitharam, M. Wang, How the beast really moves: Cayley analysis of mechanism realization spaces using caymos, Computer-Aided Design 46 (0) (2014) 205 - 210, 2013 SIAM Conference on Geometric and Physical Modeling.

[16] M. Sitharam, M. Wang, Cayley configuration space analysis by CayMos: software architecture and functionalities, In preparation.

[17] M. Wang, M. Sitharam, Cayley analysis of mechanism configuration spaces using caymos: Software functionalities and architecture, ACM Transactions on Mathematical Software.

[18] R. Joan-Arinyo, A. Soto-Riera, S. Vila-Marta, J. Vilaplana-Pastó, Transforming an under-constrained geometric constraint problem into a well-constrained one, in: Proceedings of the eighth ACM symposium on Solid modeling and applications, SM '03, ACM, New York, NY, USA, 2003, pp. 33-44. doi:10.1145/781606.781616 URL http://doi.acm.org/10.1145/781606.781616

[19] M. E. Gaspar, P. Csermely, Rigidity and flexibility of biological networks, ArXiv e-printsarXiv:1204.6389

[20] Wikimedia Commons, Chlamydomonas tem 17 https://commons.wikimedia.org/wiki/File:Chlamydomonas_TEM_17.jpg (2007).

URL https://commons.wikimedia.org/wiki/File:Chlamydomonas_TEM_17.jpg

[21] S. J. Hollister, Bme 332: Introduction to biosolid mechanics, http://www.umich.edu/ bme332/ch10ligten/ligten2.jpg (2007).

[22] Wikimedia Commons, Rothemund dna sierpinski gasket https://commons.wikimedia.org/wiki/File: Rothemund-DNA-SierpinskiGasket.jpg (2007).

URL https://commons.wikimedia.org/wiki/File:Rothemund-DNA-SierpinskiGasket.jpg

[23] D. J. Needleman, M. A. Ojeda-Lopez, U. Raviv, H. P. Miller, L. Wilson, C. R. Safinya, Higher-order assembly of microtubules by counterions: From hexagonal bundles to living necklaces, Proceedings of the National Academy of Sciences of 
the United States of America 101 (46) (2004) 16099-16103. arXiv:http://www.pnas.org/content/101/46/16099.full. pdfthtml doi:10.1073/pnas.0406076101

URL http://www .pnas .org/content/101/46/16099.abstract

[24] F. R. Garcia-Gonzalo, J. F. Reiter, Scoring a backstage pass: Mechanisms of ciliogenesis and ciliary access The Journal of Cell Biology 197 (6) (2012) 697-709. arXiv:http://jcb.rupress.org/content/197/6/697.full.pdf+html, doi:10.1083/ jcb.201111146.

URL http://jcb.rupress.org/content/197/6/697.abstract

[25] Wikimedia Commons, Afm height image of carboxymethylated nanocellulose adsorbed on a silica surface. https:// commons.wikimedia.org/wiki/File:AFM_Innventia_nanocellulose.JPG (2010)

URL https://commons.wikimedia.org/wiki/File:AFM_Innventia_nanocellulose.JPG

[26] Wikimedia Commons, Plant cell wall diagram, https://commons.wikimedia.org/wiki/File:Plant_cell_wall_diagram. svg (2007).

URL https://commons.wikimedia.org/wiki/File:Plant_cell_wall_diagram.svg

[27] T. Björkman, S. Kurasch, O. Lehtinen, J. Kotakoski, O. V. Yazyev, A. Srivastava, V. Skakalova, J. H. Smet, U. Kaiser, A. V. Krasheninnikov, Defects in bilayer silica and graphene: common trends in diverse hexagonal two-dimensional systems Scientific Reports 3 (3482). doi:10.1038/srep03482

URL http://dx.doi.org/10.1038/srep03482

[28] F. R. Eder, J. Kotakoski, U. Kaiser, J. C. Meyer, A journey from order to disorder - Atom by atom transformation from graphene to a 2D carbon glass, Scientific Reports 4 (4060). doi:10.1038/srep04060 URL http://dx.doi.org/10.1038/srep04060

[29] P. W. K. Rothemund, N. Papadakis, E. Winfree, Algorithmic self-assembly of dna sierpinski triangles, PLoS Biology 2 (12). doi:10.1371/journal.pbio.0020424

[30] Wikimedia Commons, Finite subdivision of a radial link https://commons.wikimedia.org/wiki/File:Finite_ subdivision_of_a_radial_link.png (2012).

URL https://commons.wikimedia.org/wiki/File:Finite_subdivision_of_a_radial_link.png

[31] M. Wilson, A. Kumar, D. Sherrington, M. F. Thorpe, Modeling vitreous silica bilayers, Physical Review B 87 (21) (2013) 214108. arXiv:1303.5898, doi:10.1103/PhysRevB.87.214108

[32] M. Heyde, Structure and motion of a 2d glass Science 342 (6155) (2013) 201-202. arXiv:http://www.sciencemag.org/ content/342/6155/201.full.pdf doi:10.1126/science.1245217

URL http://www.sciencemag.org/content/342/6155/201.short

[33] A. Donev, S. Torquato, F. H. Stillinger, R. Connelly, Jamming in hard sphere and disk packings, Journal of Applied Physics 95 (3)

[34] X.-S. Gao, Q. Lin, G.-F. Zhang, A c-tree decomposition algorithm for 2d and 3d geometric constraint solving, Comput. Aided Des. 38 (1) (2006) 1-13. doi:10.1016/j.cad.2005.03.002

URL http://dx.doi.org/10.1016/j.cad.2005.03.002

[35] B. Jackson, T. Jordán, Pin-collinear body-and-pin frameworks and themolecular conjecture, Discrete \& Computational Geometry 40 (2) (2008) 258-278. doi:10.1007/s00454-008-9100-z URL http://dx.doi.org/10.1007/s00454-008-9100-z

[36] M. Sitharam, M. Tarifi, M. Wang, An incidence geometry approach to dictionary learning, in: Proceedings of CCCG 2014, 2014 .

[37] L. Asimow, B. Roth, The rigidity of graphs, Transactions of the American Mathematical Society 245 (1978) $279-289$. doi:http://dx.doi.org/10.1090/S0002-9947-1978-0511410-9

[38] L. Lovasz, Y. Yemini, On generic rigidity in the plane, SIAM Journal on Algebraic Discrete Methods 3 (1) (1982) 91-98.

[39] A. Lee, I. Streinu, L. Theran, Graded sparse graphs and matroids., J. UCS 13 (11) (2007) 1671-1679.

[40] D. J. Jacobs, B. Hendrickson, An algorithm for two-dimensional rigidity percolation: The pebble game, Journal of Computational Physics 137 (2) (1997) 346-365. doi:http://dx.doi.org/10.1006/jcph.1997.5809 URL http://www.sciencedirect.com/science/article/pii/S0021999197958095

[41] C. M. Hoffmann, A. Lomonosov, M. Sitharam, Finding solvable subsets of constraint graphs, in: G. Smolka (Ed.), Principles and Practice of Constraint Programming-CP97, Vol. 1330 of Lecture Notes in Computer Science, Springer Berlin Heidelberg, 1997, pp. 463-477. doi:10.1007/BFb0017460. URL http://dx.doi.org/10.1007/BFb0017460

[42] C. Jermann, G. Trombettoni, B. Neveu, P. Mathis, Decomposition of geometric constraint systems: a survey, International Journal of Computational Geometry \& Applications 16 (05n06) (2006) 379-414.

[43] A. Lee, I. Streinu, Pebble Game Algorithms and Sparse Graphs, ArXiv Mathematics e-printsarXiv:math/0702129

[44] J. C. Owen, Algebraic solution for geometry from dimensional constraints in: Proceedings of the First ACM Symposium on Solid Modeling Foundations and CAD/CAM Applications, SMA '91, ACM, New York, NY, USA, 1991 , pp. $397-407$. doi:10.1145/112515.112573

URL http://doi.acm.org/10.1145/112515.112573

[45] I. Fudos, C. M. Hoffmann, A graph-constructive approach to solving systems of geometric constraints ACM Trans. Graph. 16 (2) (1997) 179-216. doi:10.1145/248210.248223 URL http://doi.acm.org/10.1145/248210.248223

[46] R. Joan-Arinyo, A. Soto-Riera, S. Vila-Marta, J. Vilaplana-Pastó, Revisiting decomposition analysis of geometric constraint graphs, Computer-Aided Design 36 (2) (2004) 123 - 140, solid Modeling and Applications. doi:http: //dx.doi.org/10.1016/S0010-4485(03)00057-5 URL http://www.sciencedirect.com/science/article/pii/S0010448503000575 
[47] J. Saxe, Embeddability of weighted graphs in k-space is strongly np-hard, in: Proceedings of 17th Allerton Conference in Communications, Control and Computing, 1979, pp. 480-489.

[48] C. Borcea, I. Streinu, The number of embeddings of minimally rigid graphs, Discrete \& Computational Geometry 31 (2) (2004) 287-303. doi:10.1007/s00454-003-2902-0

[49] M. Sitharam, A. Arbree, Y. Zhou, N. Kohareswaran, Solution space navigation for geometric constraint systems ACM Trans. Graph. 25 (2) (2006) 194-213. doi:10.1145/1138450.1138452 URL http://doi.acm.org/10.1145/1138450.1138452

[50] M. R. Hidalgo, R. Joan-Arinyo, The reachability problem in constructive geometric constraint solving based dynamic geometry, Journal of Automated Reasoning (2011) 1-24.

[51] Key Curriculum, The geometer's sketchpad, http://www.keycurriculum.com/ (1995).

[52] J. M. Porta, L. Ros, O. Bohigas, M. Manubens, C. Rosales, L. Jaillet, An open-source toolbox for motion analysis of closed-chain mechanisms, in: Computational Kinematics, Springer, 2014, pp. 147-154, cUIK.

[53] Siemens, D-cubed, http://www.plm.automation.siemens.com/en_us/products/open/d-cubed/ (1999).

[54] P. Todd, Geometry expressions: A constraint based interactive symbolic geometry system, in: Automated Deduction in Geometry, Springer, 2007, pp. 189-202.

[55] J. Valdes, R. E. Tarjan, E. L. Lawler, The recognition of series parallel digraphs, in: Proceedings of the eleventh annual ACM symposium on Theory of computing, ACM, 1979, pp. 1-12.

56] G.-F. Zhang, X.-S. Gao, Well-constrained completion and decomposition for under-constrained geometric constraint problems, International Journal of Computational Geometry \& Applications 16 (05n06) (2006) 461-478.

[57] Wikimedia Commons, Silica https://commons.wikimedia.org/wiki/File:Silica.svg (2008). URL https://commons .wikimedia.org/wiki/File:Silica.svg

[58] Wikimedia Commons, Tetrahedral 3d balls https://commons.wikimedia.org/wiki/File:Tetrahedral-3D-balls.png (2006). URL https://commons .wikimedia.org/wiki/File:Tetrahedral-3D-balls.png

[59] J. W. Canon, W. J. Floyd, W. R. Parry, Finite subdivision rules, Conform. Geom. Dyn. (2001) $153-196$.

[60] M. J. Buehler, Nanomechanics of collagen fibrils under varying cross-link densities: atomistic and continuum studies, Journal of the mechanical behavior of biomedical materials 1 (1) (2008) 59-67.

[61] A. B. Fall, S. B. Lindström, J. Sprakel, L. Wågberg, A physical cross-linking process of cellulose nanofibril gels with shear-controlled fibril orientation, Soft Matter 9 (6) (2013) 1852-1863.

[62] G. Smith, Plant cell wall structure and cell wall growth, Tuatara 19 (1971) 43-50.

[63] J. E. Graver, B. Servatius, H. Servatius, Combinatorial rigidity Graduate studies in mathematics, American mathematical society, Providence (R.I.), 1993. URL http://opac.inria.fr/record=b1131810

[64] L. Cai, F. Maffray, On the \{SPANNING\} k-tree problem, Discrete Applied Mathematics 44 (1-3) (1993) 139 - 156. doi:http://dx.doi.org/10.1016/0166-218X(93)90228-G URL http://www.sciencedirect.com/science/article/pii/0166218X9390228G

[65] B. Servatius, On the rigidity of ramanujan graphs, Ann. Univ. Sci. Budapest. Eötvös Sect. Math 43 (2000) 165-170. 